\title{
The Synthesis of Non-Racemic Allylic Hydroxy Phosphonates via Alkene Cross Metathesis
}

Anyu He, Bingli Yan, Anchalee Thanavaro, Christopher D. Spilling, * and Nigam P. Rath

Department of Chemistry and Biochemistry, University of Missouri-St. Louis, 8001 Natural Bridge Road, St. Louis, Missouri 63121, USA.

cspill@umsl.edu

\section{Supporting Information}

Table of Contents

General Experimental

S3

${ }^{1} \mathrm{H}$ and ${ }^{13} \mathrm{C}$ NMR spectrum for $\mathbf{4 a}$

S4

${ }^{1} \mathrm{H}$ and ${ }^{13} \mathrm{C}$ NMR spectrum for $5 \mathbf{a}$

S5

${ }^{1} \mathrm{H}$ and ${ }^{13} \mathrm{C}$ NMR spectrum for $\mathbf{6 a}$

S6

${ }^{1} \mathrm{H}$ and ${ }^{13} \mathrm{C}$ NMR spectrum for $7 \mathbf{a}$

S7

${ }^{1} \mathrm{H}$ and ${ }^{13} \mathrm{C}$ NMR spectrum for 8

S8

${ }^{1} \mathrm{H}$ and ${ }^{13} \mathrm{C}$ NMR spectrum for $\mathbf{2 d}$

S9

${ }^{1} \mathrm{H}$ and ${ }^{13} \mathrm{C}$ NMR spectrum for $\mathbf{2 e}$

${ }^{1} \mathrm{H}$ and ${ }^{13} \mathrm{C}$ NMR spectrum for $\mathbf{2 f}$

${ }^{1} \mathrm{H}$ and ${ }^{13} \mathrm{C}$ NMR spectrum for $2 \mathrm{~g}$

$\mathrm{S} 12$

${ }^{1} \mathrm{H}$ and ${ }^{13} \mathrm{C}$ NMR spectrum for $\mathbf{3 d}$

${ }^{1} \mathrm{H}$ and ${ }^{13} \mathrm{C}$ NMR spectrum for $4 d$

${ }^{1} \mathrm{H}$ and ${ }^{13} \mathrm{C}$ NMR spectrum for $\mathbf{5 d}$ 
${ }^{1} \mathrm{H}$ and ${ }^{13} \mathrm{C}$ NMR spectrum for $\mathbf{6 d} \quad \mathrm{S} 16$

$\begin{array}{ll}{ }^{1} \mathrm{H} \text { and }{ }^{13} \mathrm{C} \text { NMR spectrum for } \mathbf{7 d} & \text { S17 }\end{array}$

${ }^{1} \mathrm{H}$ and ${ }^{13} \mathrm{C}$ NMR spectrum for $\mathbf{3 e} \quad \mathrm{S} 18$

$\begin{array}{ll}{ }^{1} \mathrm{H} \text { and }{ }^{13} \mathrm{C} \text { NMR spectrum for } \mathbf{3 g} & \mathrm{S} 19\end{array}$

$\begin{array}{ll}{ }^{1} \mathrm{H} \text { and }{ }^{13} \mathrm{C} \text { NMR spectrum for } \mathbf{3 h} & \text { S20 }\end{array}$

${ }^{1} \mathrm{H}$ and ${ }^{13} \mathrm{C}$ NMR spectrum for $\mathbf{3 i} \quad$ S21

${ }^{1} \mathrm{H}$ and ${ }^{13} \mathrm{C}$ NMR spectrum for $\mathbf{3 k} \quad \quad$ S22

${ }^{1} \mathrm{H}$ and ${ }^{13} \mathrm{C}$ NMR spectrum for $\mathbf{3 l} \quad$ S23

${ }^{1} \mathrm{H}$ and ${ }^{13} \mathrm{C}$ NMR spectrum for $\mathbf{3 m} \quad \mathrm{S} 24$

$\begin{array}{ll}{ }^{1} \mathrm{H} \text { and }{ }^{13} \mathrm{C} \text { NMR spectrum for } \mathbf{3 n} & \text { S25 }\end{array}$

${ }^{1} \mathrm{H}$ and ${ }^{13} \mathrm{C}$ NMR spectrum for $30 \quad$ S26

$\begin{array}{ll}{ }^{31} \mathrm{P} \text { NMR spectrum for the reaction of } \mathbf{2 c} \text { with allyl acetate } & \text { S27 }\end{array}$

$\begin{array}{ll}\text { HPLC data for compound } \mathbf{2 b} & \text { S28 }\end{array}$

$\begin{array}{lr}\text { HPLC data for compound 2d (from 2b) } & \text { S29 }\end{array}$

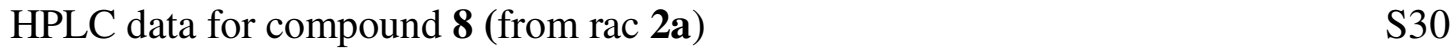

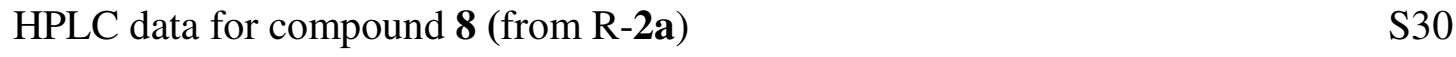

$\begin{array}{ll}\text { X-ray data tables for } 8 & \text { S32 }\end{array}$

General Experimental: ${ }^{1} \mathrm{H},{ }^{13} \mathrm{C}$ and ${ }^{31} \mathrm{P}$ NMR spectra were recorded at 300,75 and $121 \mathrm{MHz}$, repectively in $\mathrm{CDCl}_{3} .{ }^{1} \mathrm{H}$ NMR spectra are referenced to internal tetramethylsilane (TMS, $\delta=$ $0.00),{ }^{13} \mathrm{C}$ NMR spectra to the center-line of $\mathrm{CDCl}_{3}(77.23 \mathrm{ppm})$ and ${ }^{31} \mathrm{P}$ NMR spectra to external $85 \% \mathrm{H}_{3} \mathrm{PO}_{4}$. Coupling constants, $J$, are reported in $\mathrm{Hz}$. Enantiomer ratios were measured by chiral stationary-phase HPLC on a $(S, S)$-Whelk-O 1 column or a Chiralpak AS column or by ${ }^{1} \mathrm{H}$ 
NMR using Karfarski's quinine method. ${ }^{1}$ Optical rotations were determined using a polarimeter set at $589 \mathrm{~nm} . \quad( \pm)$ Dimethyl [1-hydroxy-2-propenyl] phosphonate 2a, ${ }^{2}(1 R)$ Dimethyl [1hydroxy-2-propenyl] phosphonate $(\boldsymbol{R}) \mathbf{2 a}\left(70 \%\right.$ e.e.), ${ }^{2}(1 R)(2 E)$ Dimethyl (1-hydroxy-3-phenyl2-propenyl) phosphonate $(\boldsymbol{R}) \mathbf{2 b} \quad(98 \%$ e.e. $),^{2,3,4}( \pm)$ (2E) Dimethyl (1-hydroxy-2-butenyl) phosphonate 2cc, ${ }^{4}$ Dimethyl [1-(methoxycarbonyloxy)-2-propenyl] phosphonate $\mathbf{3 a},{ }^{2}$ (2E) Dimethyl [1-(methoxycarbonyloxy)-3-phenyl-2-propenyl] phosphonate $\mathbf{3 b},{ }^{2}$ and (2E) Dimethyl [1-(methoxycarbonyloxy)-2-butenyl] phosphonate $( \pm) \mathbf{3} \mathbf{c}^{4}$ were prepared using previously published procedures.

1. Zymanczyk-Duda, E.; Skwarczynski, M.; Lejczak, B.; Karfarski, P. Tetrahedron: Asymmetry 1996, 7, 1277.

2. Yan, B.; Spilling, C. D. J. Org. Chem. 2004, 69, 2859.

3. Rowe, B. J.; Spilling, C. D. Tetrahedron: Asymmetry 2001, 12, 1701; Blazis, V. J.; Koeller, K. J.; Spilling, C. D. J. Org. Chem. 1995, 60, 931.

4. Rowe, B. J.; Spilling, C. D. J. Org. Chem. 2003, 68, 9502 

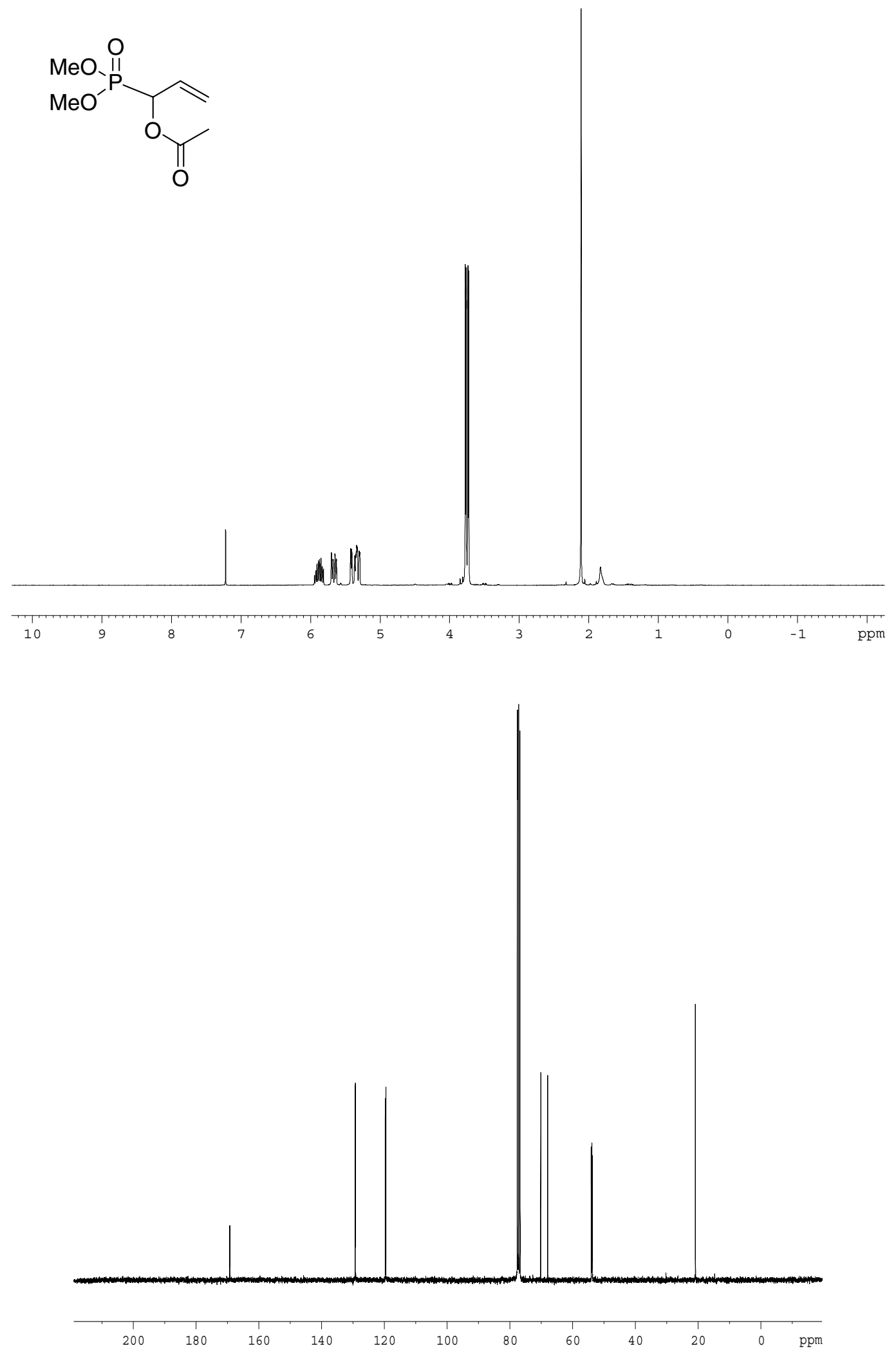

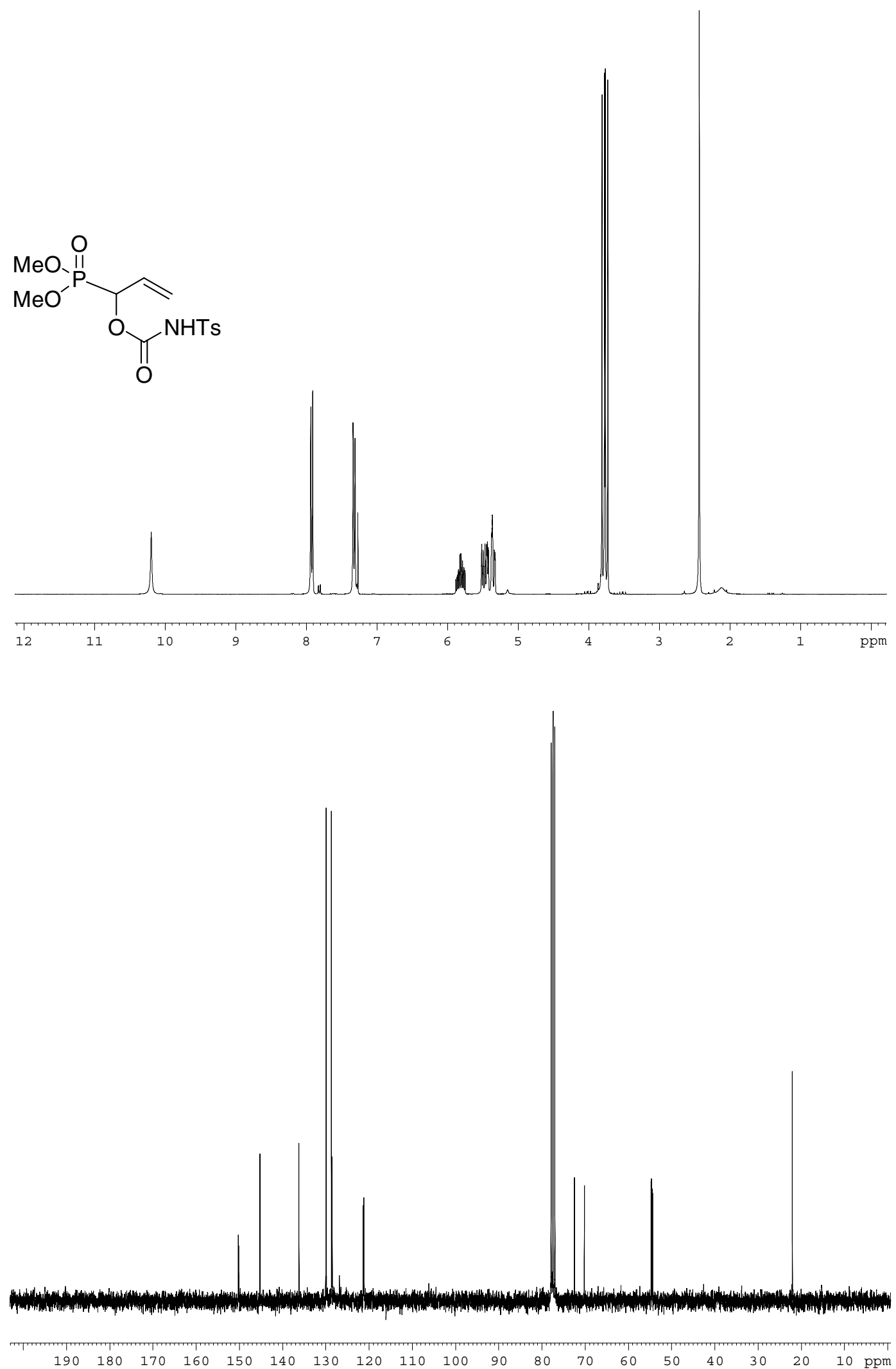

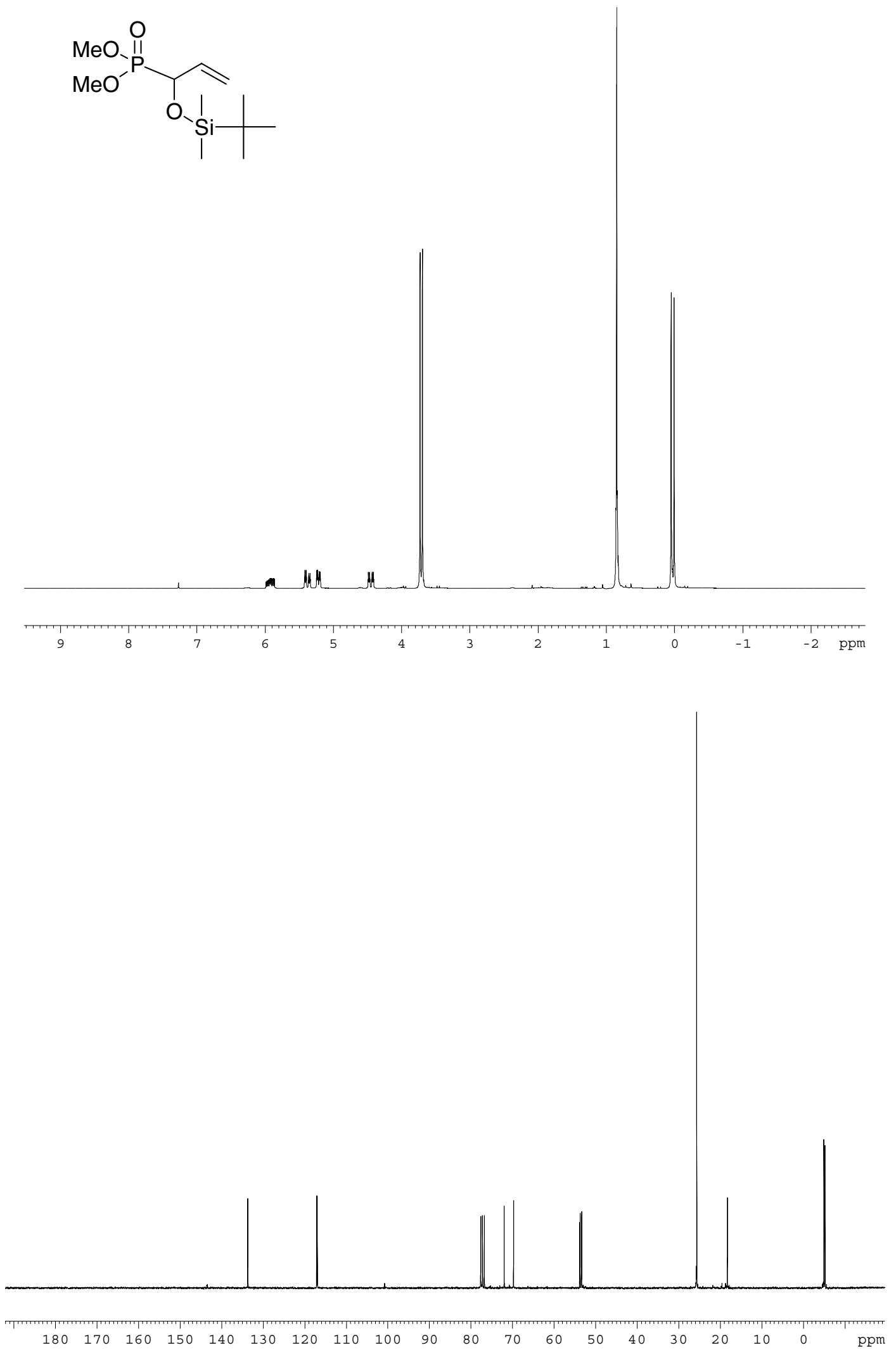


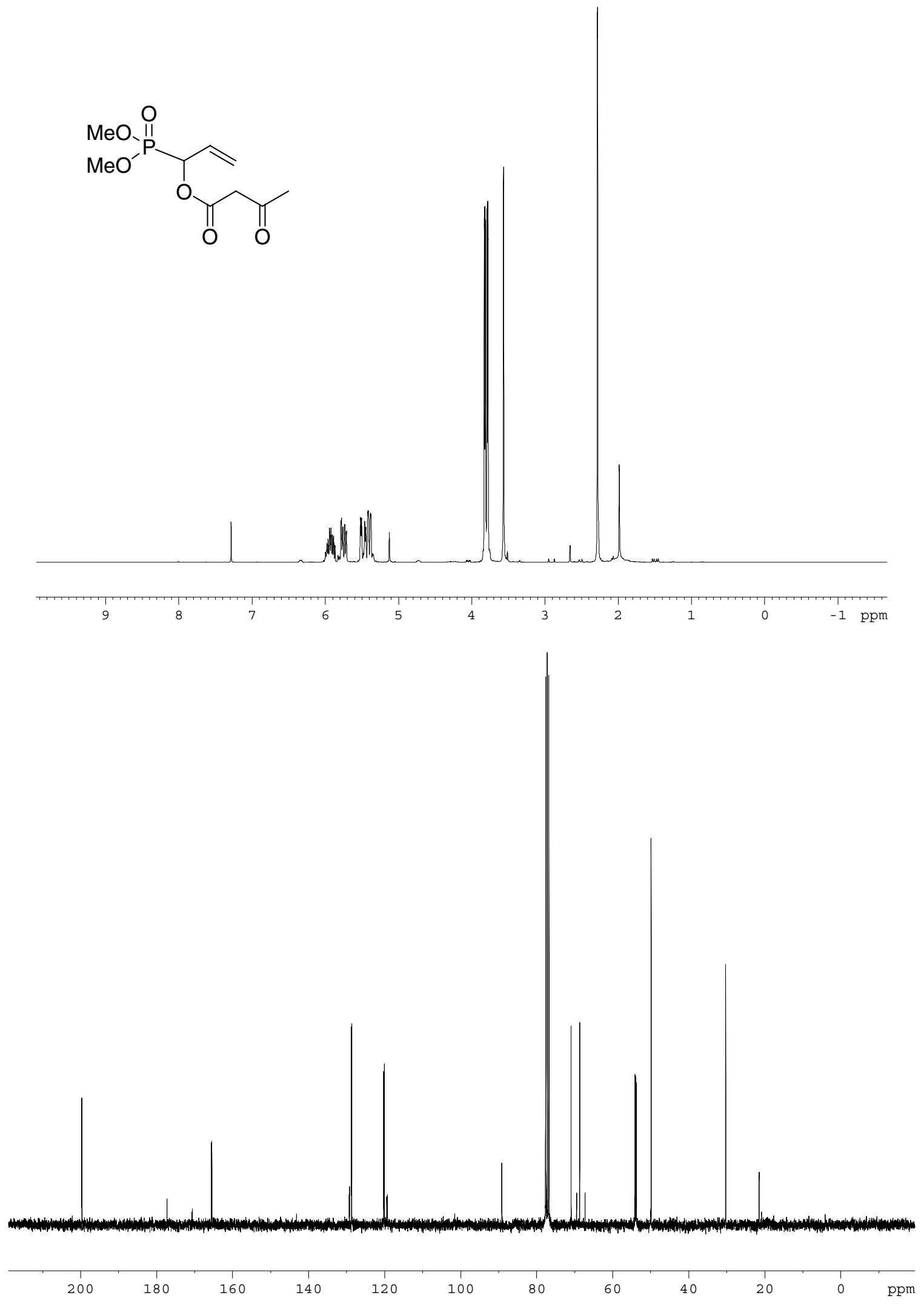



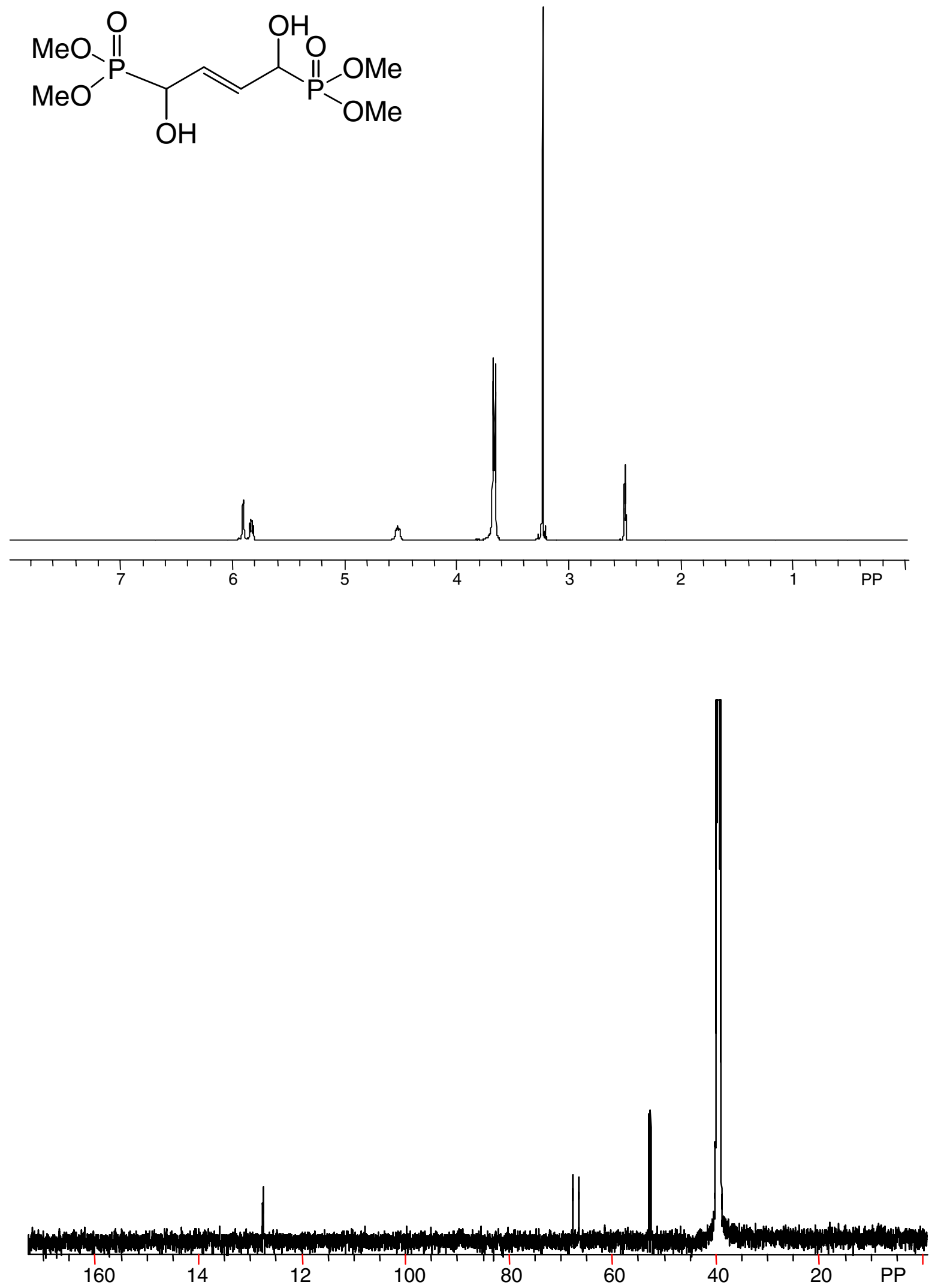


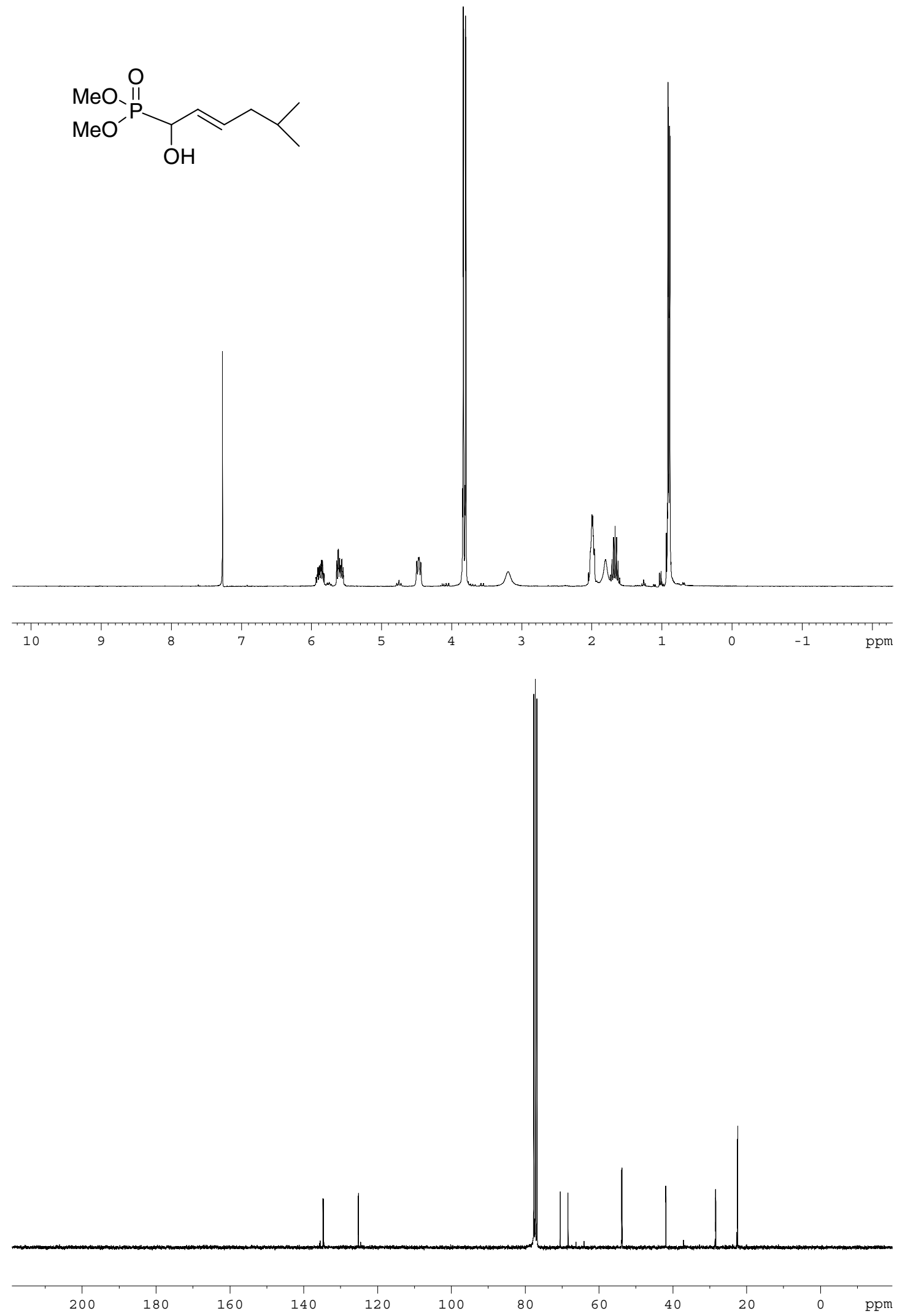



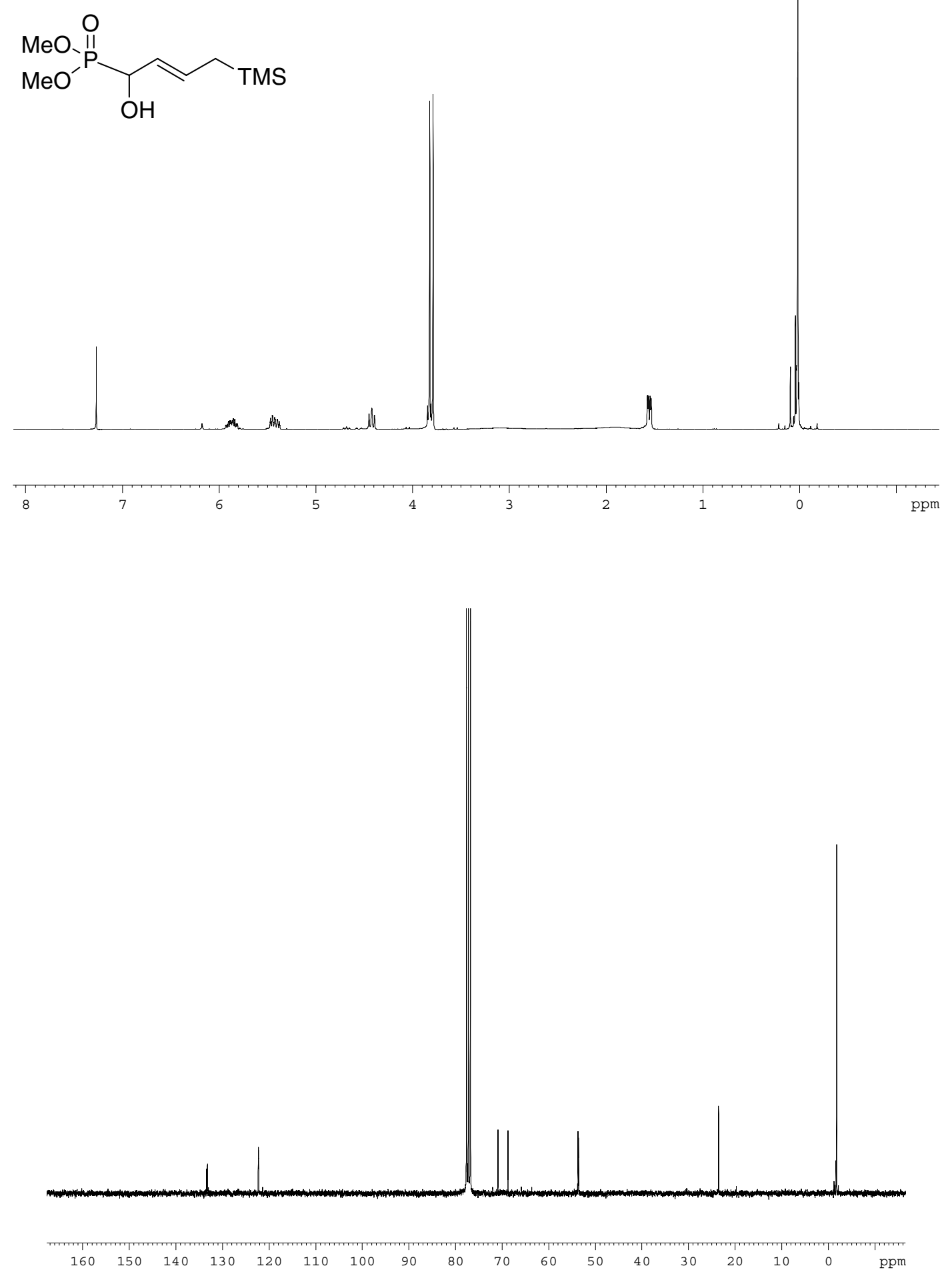

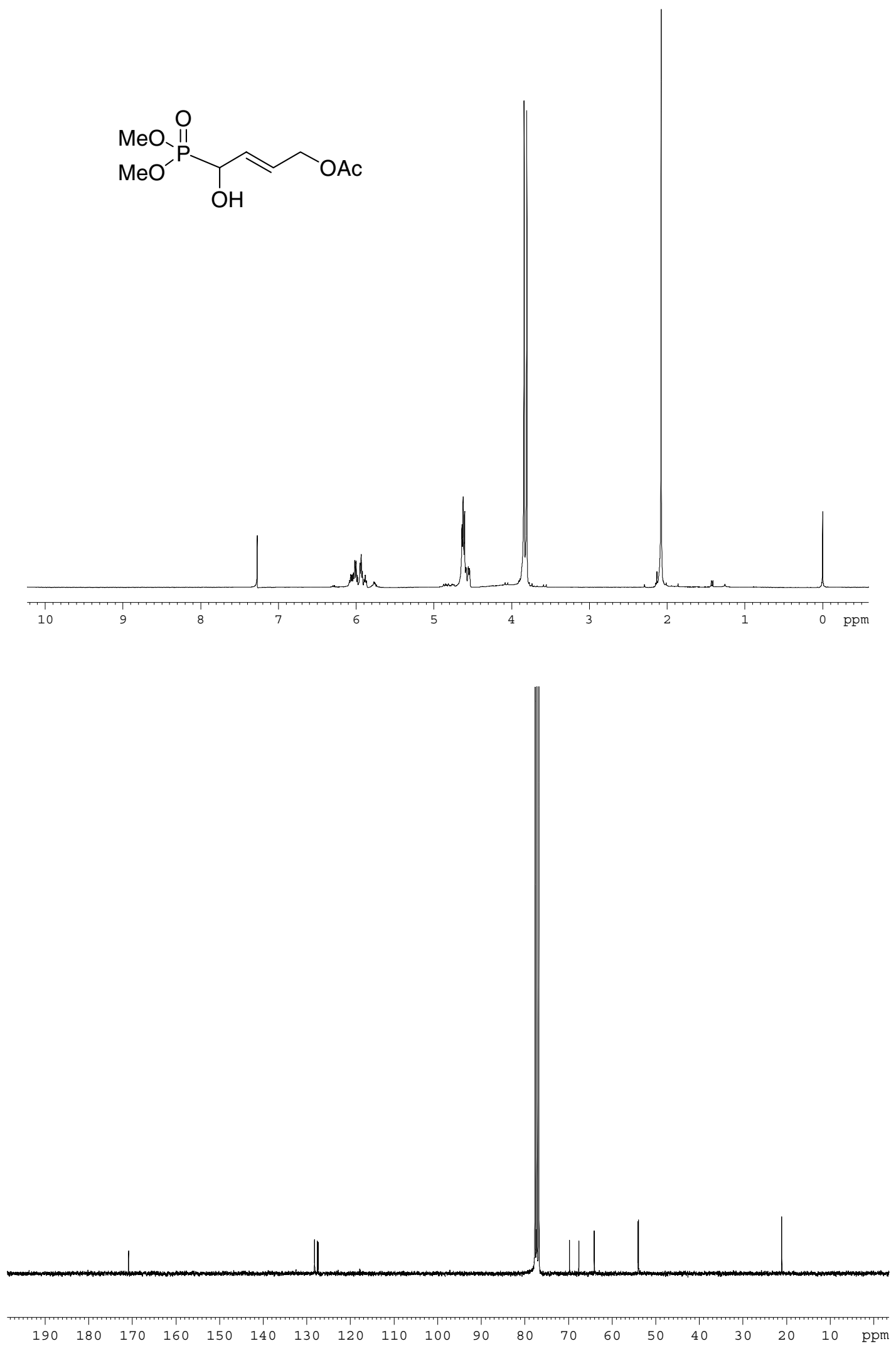

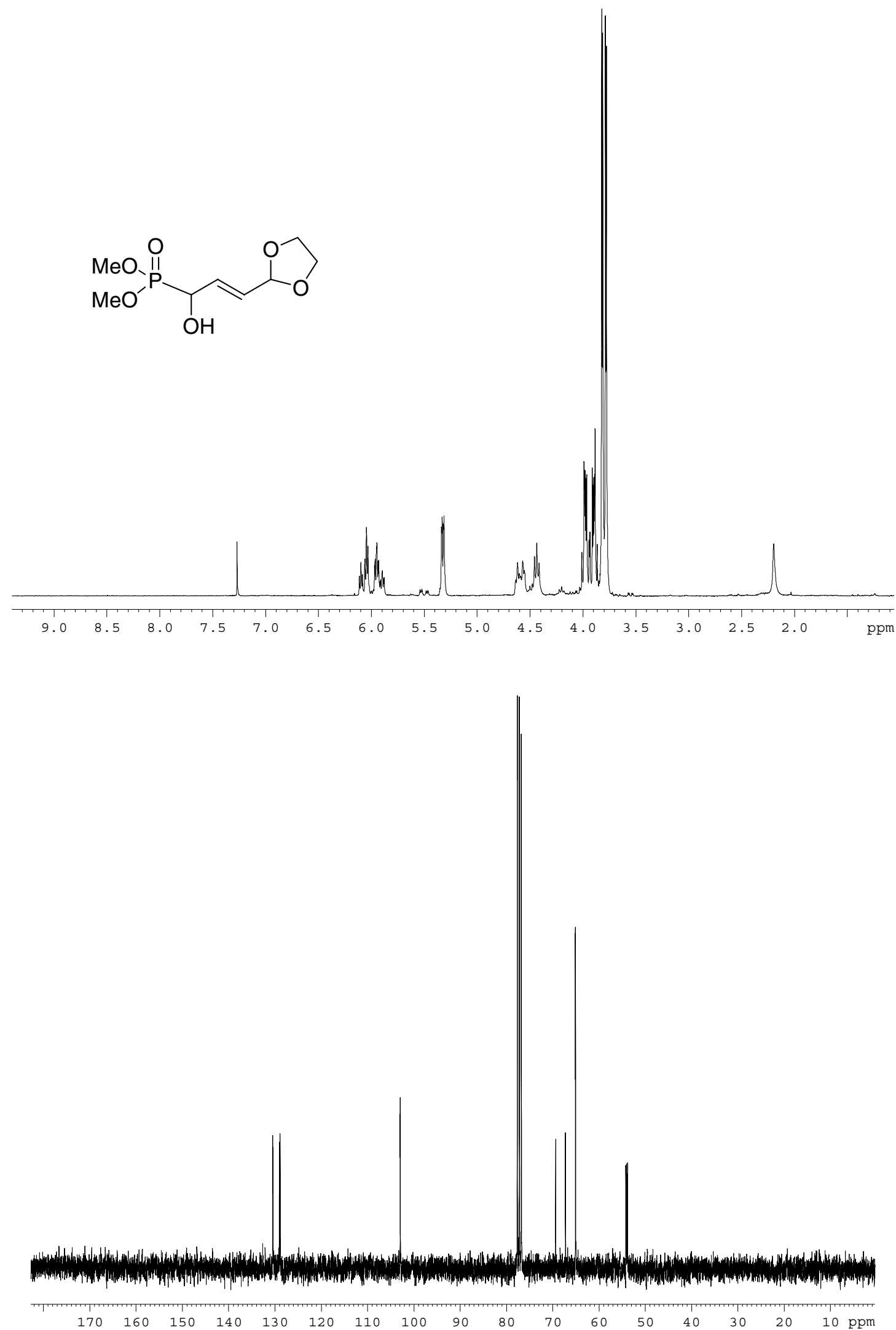

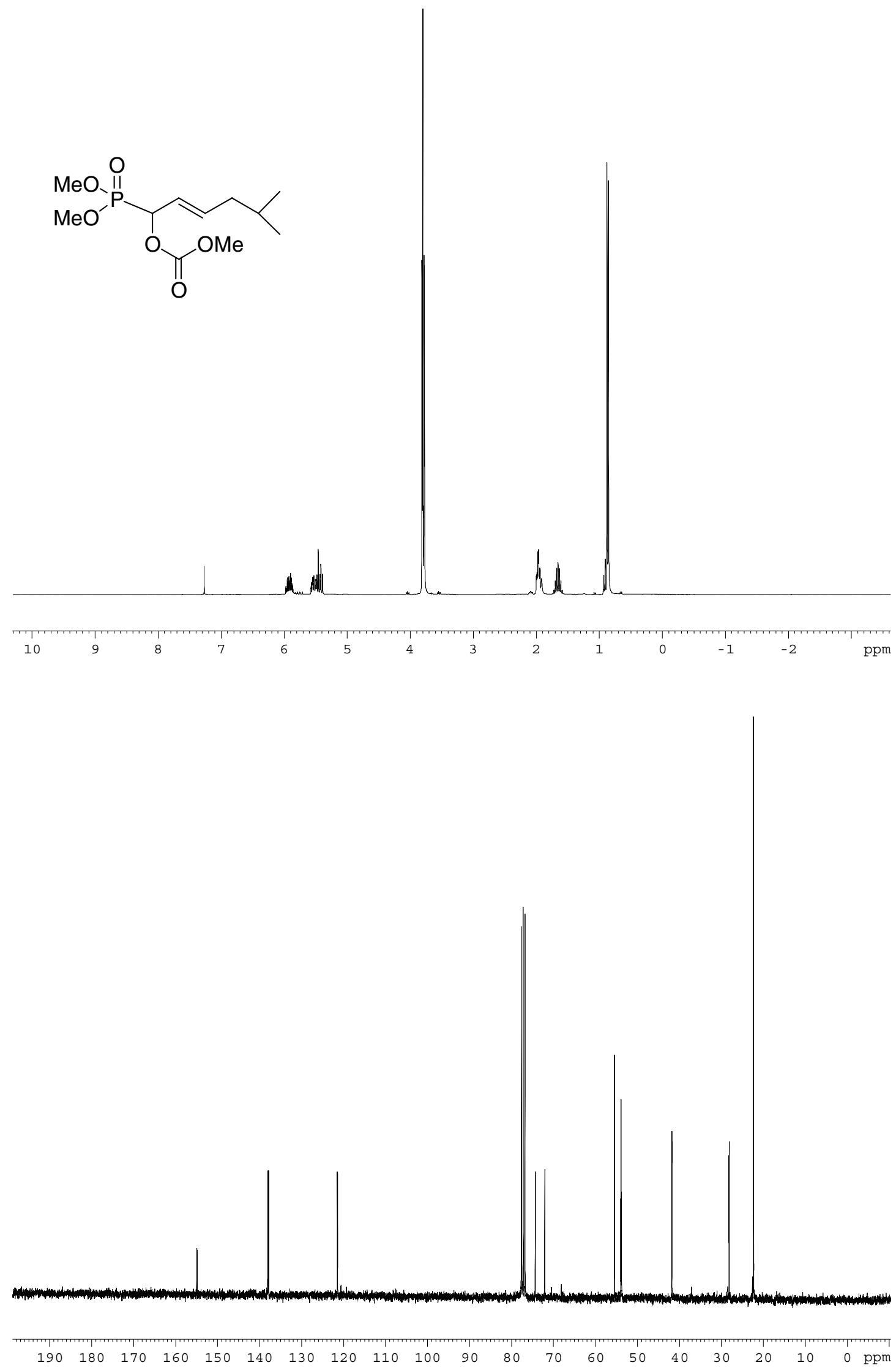

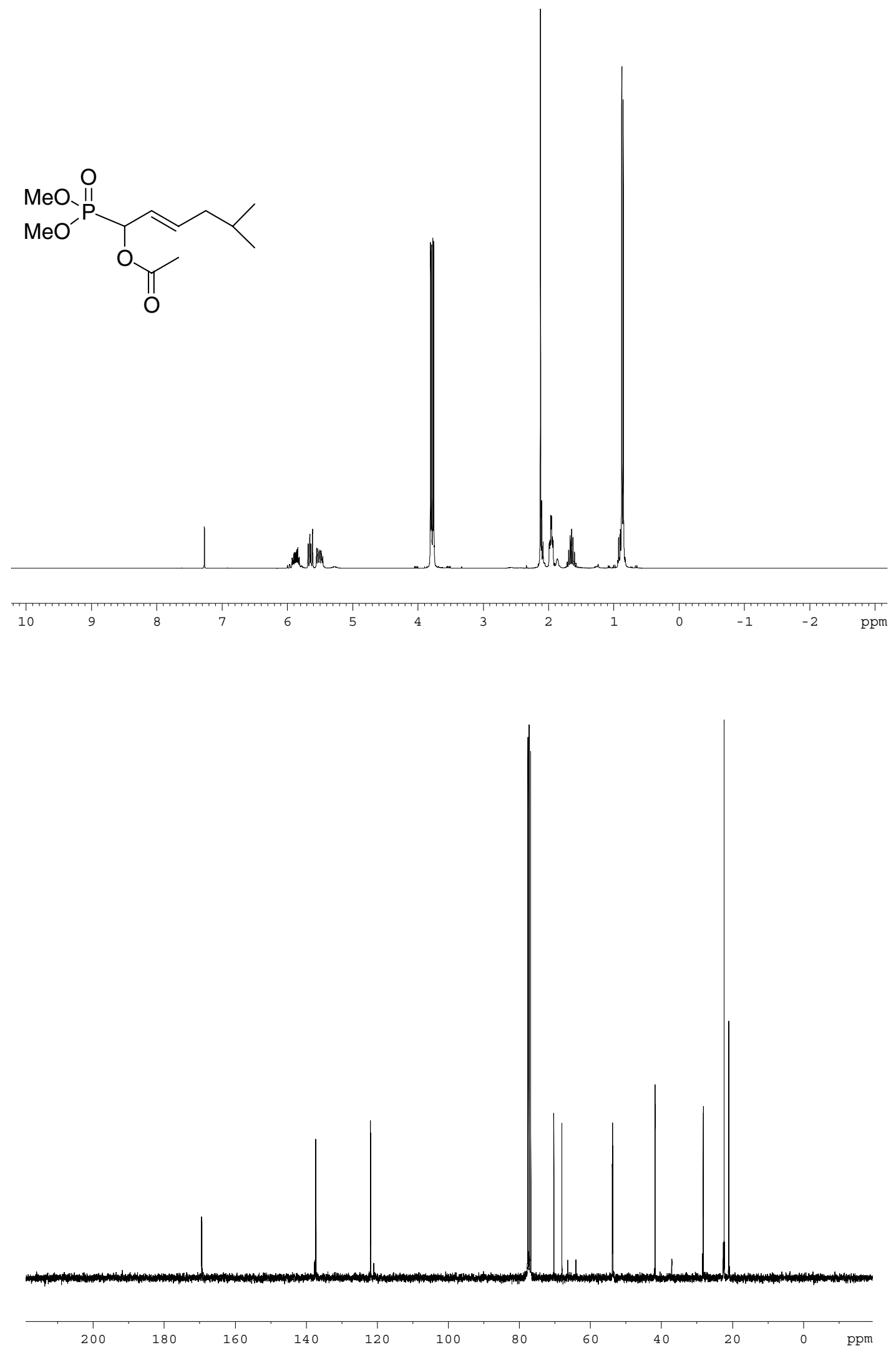

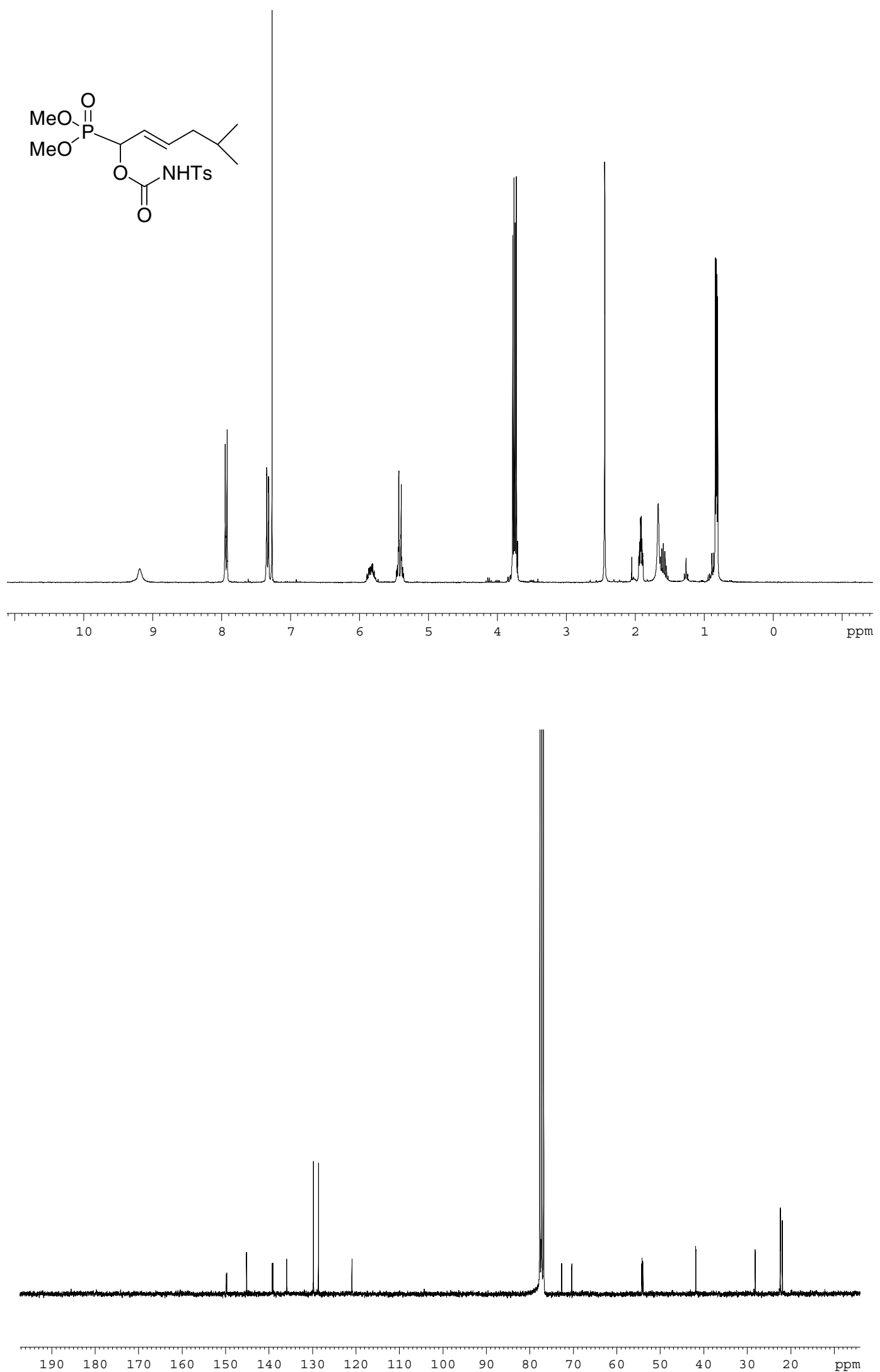


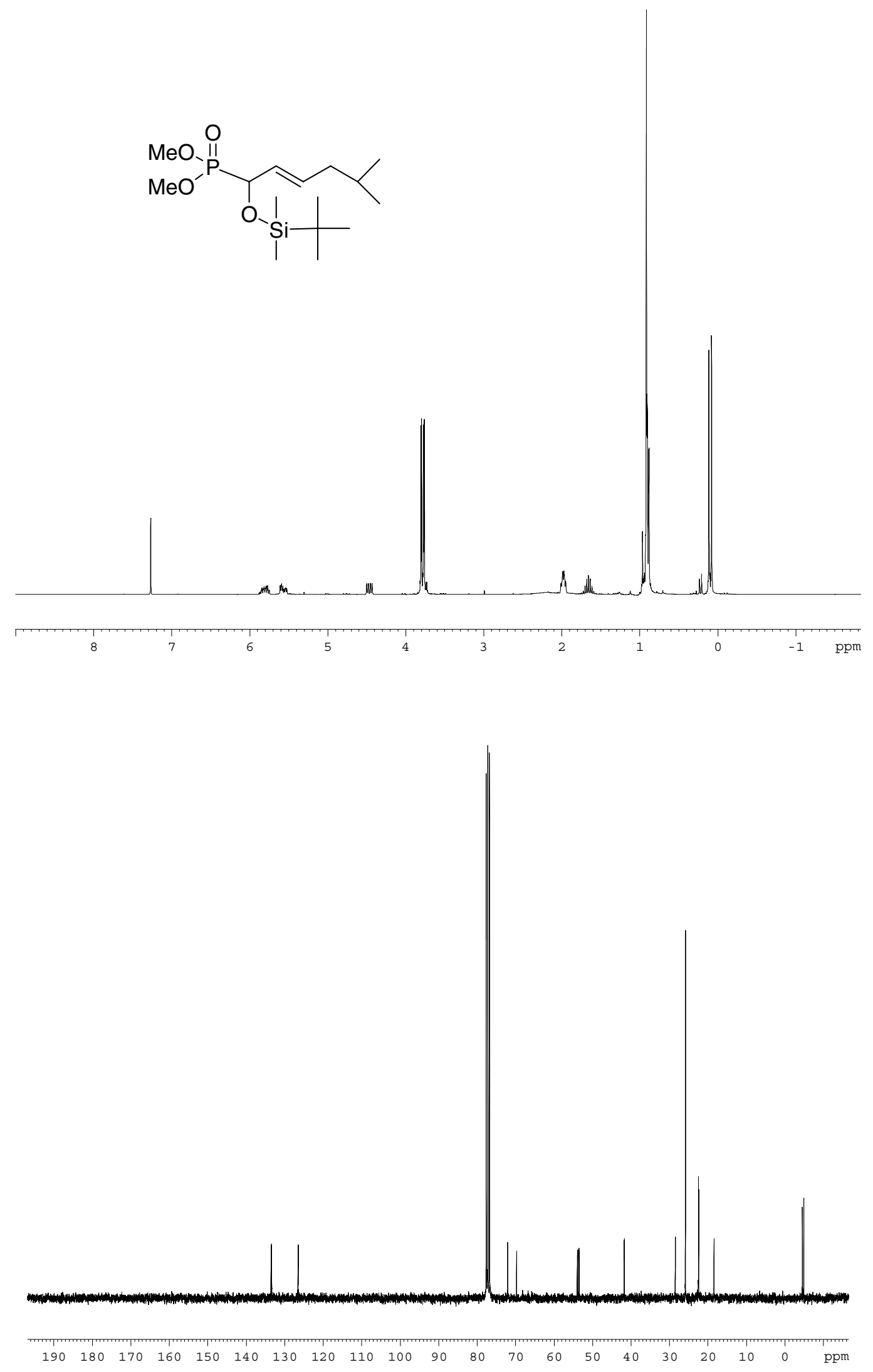




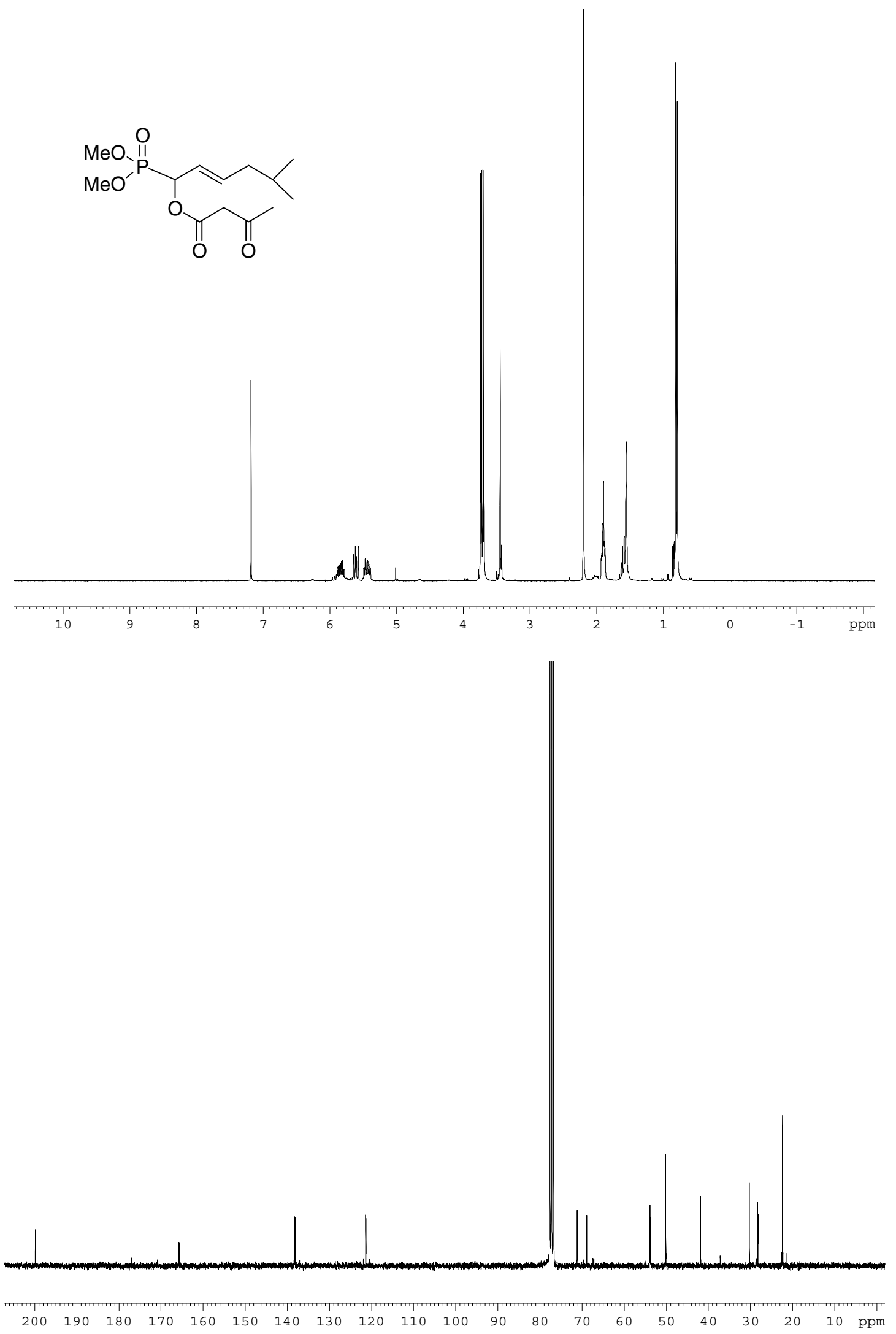



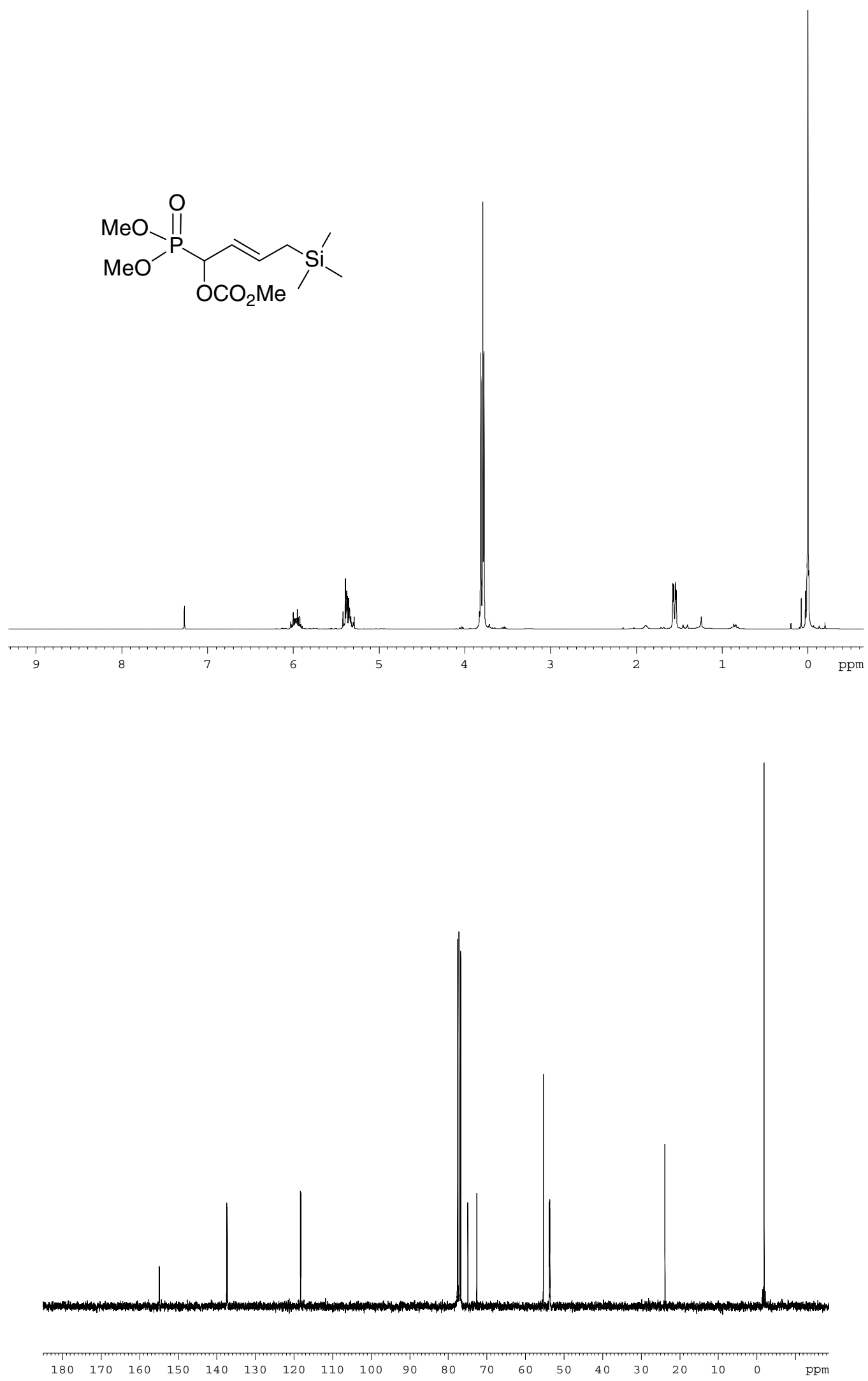

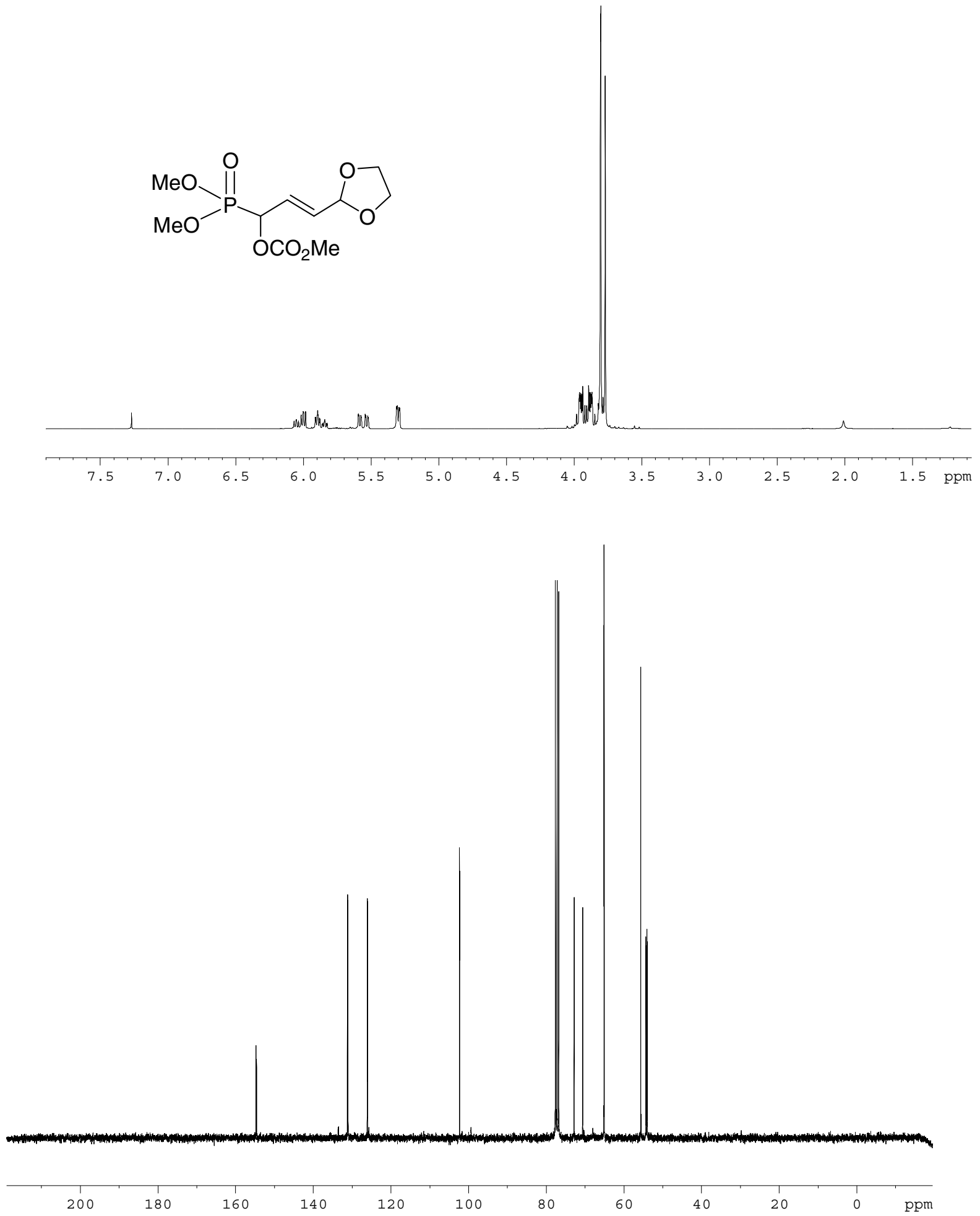


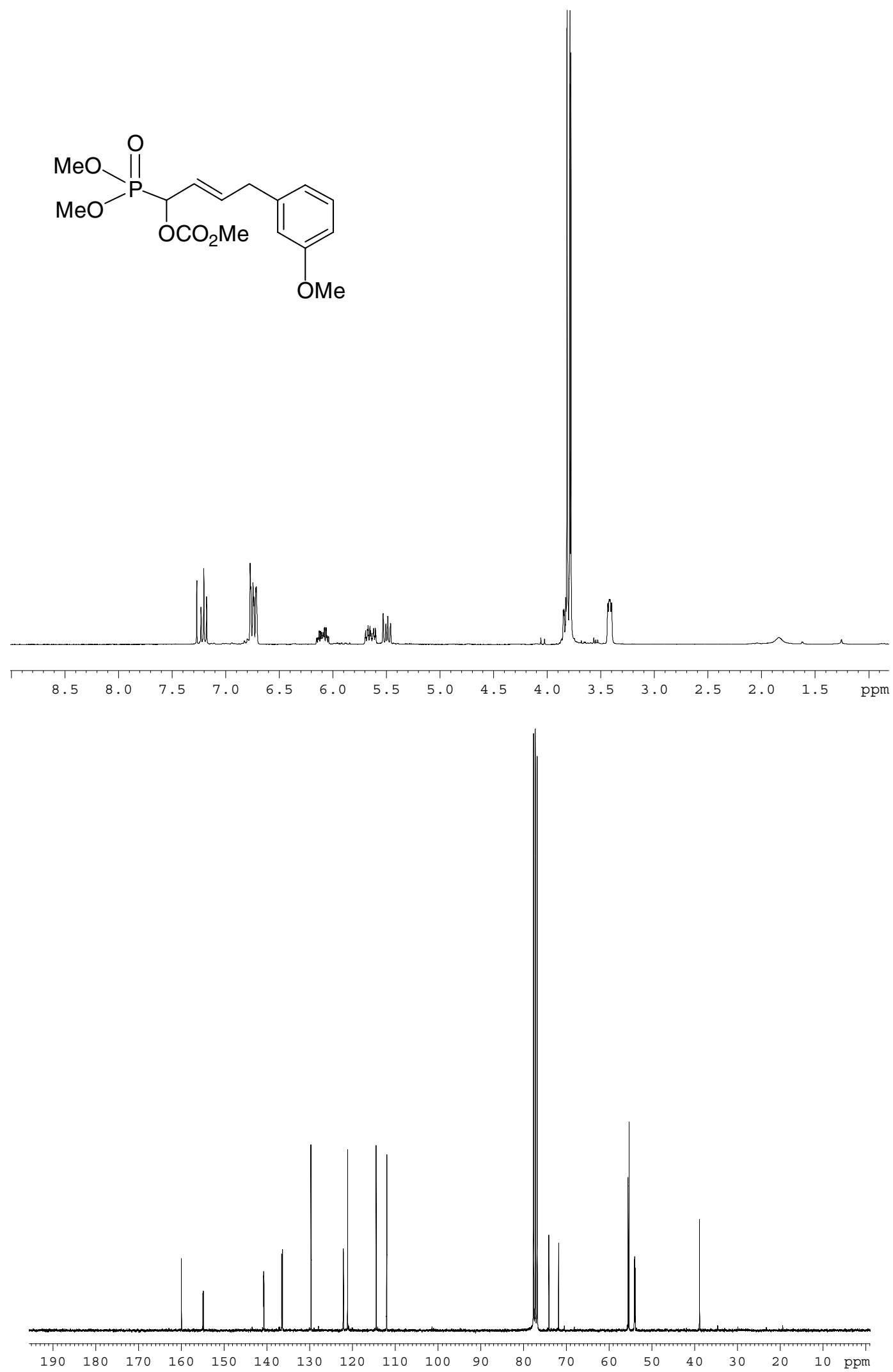



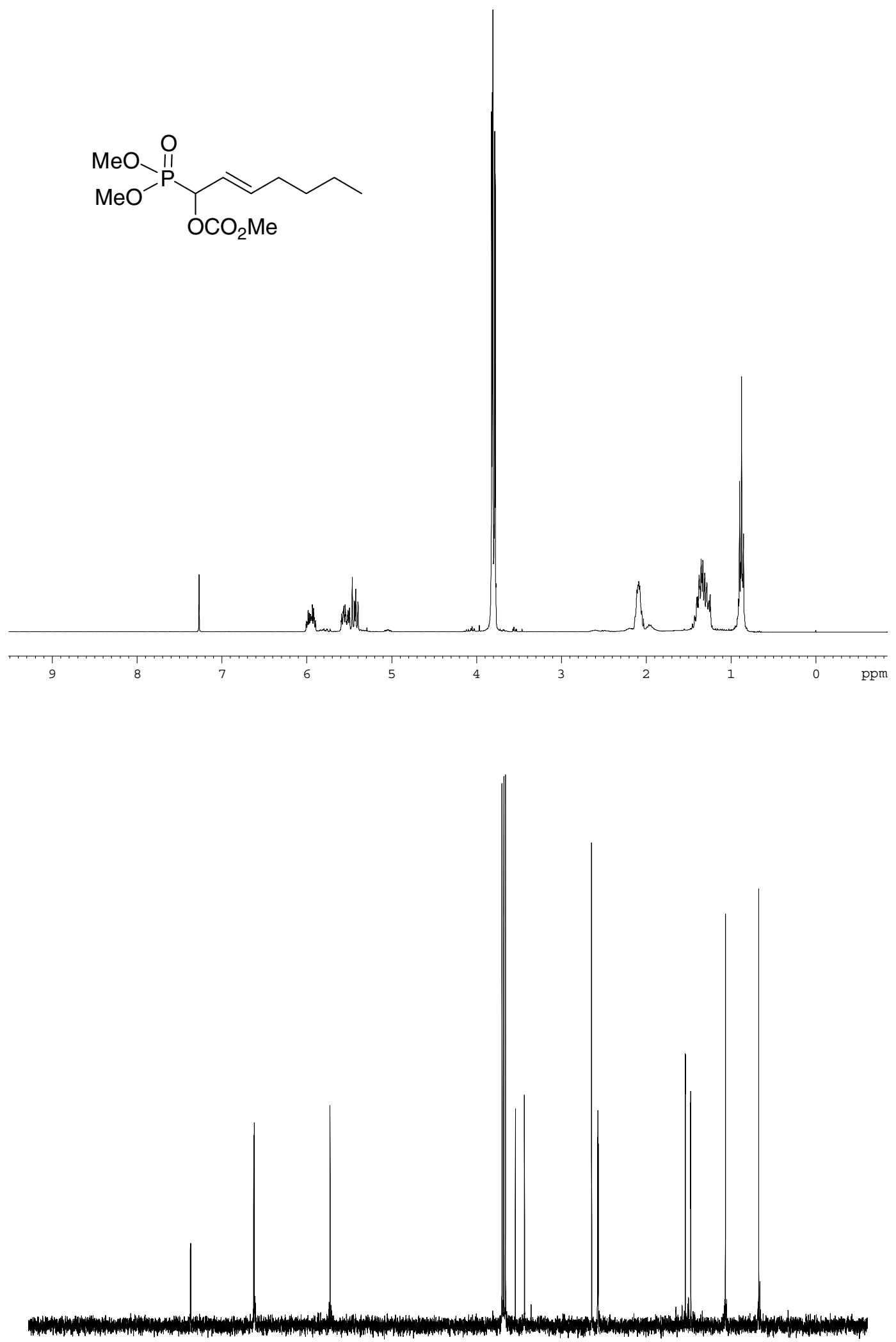

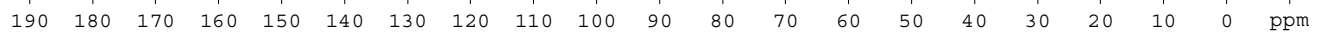



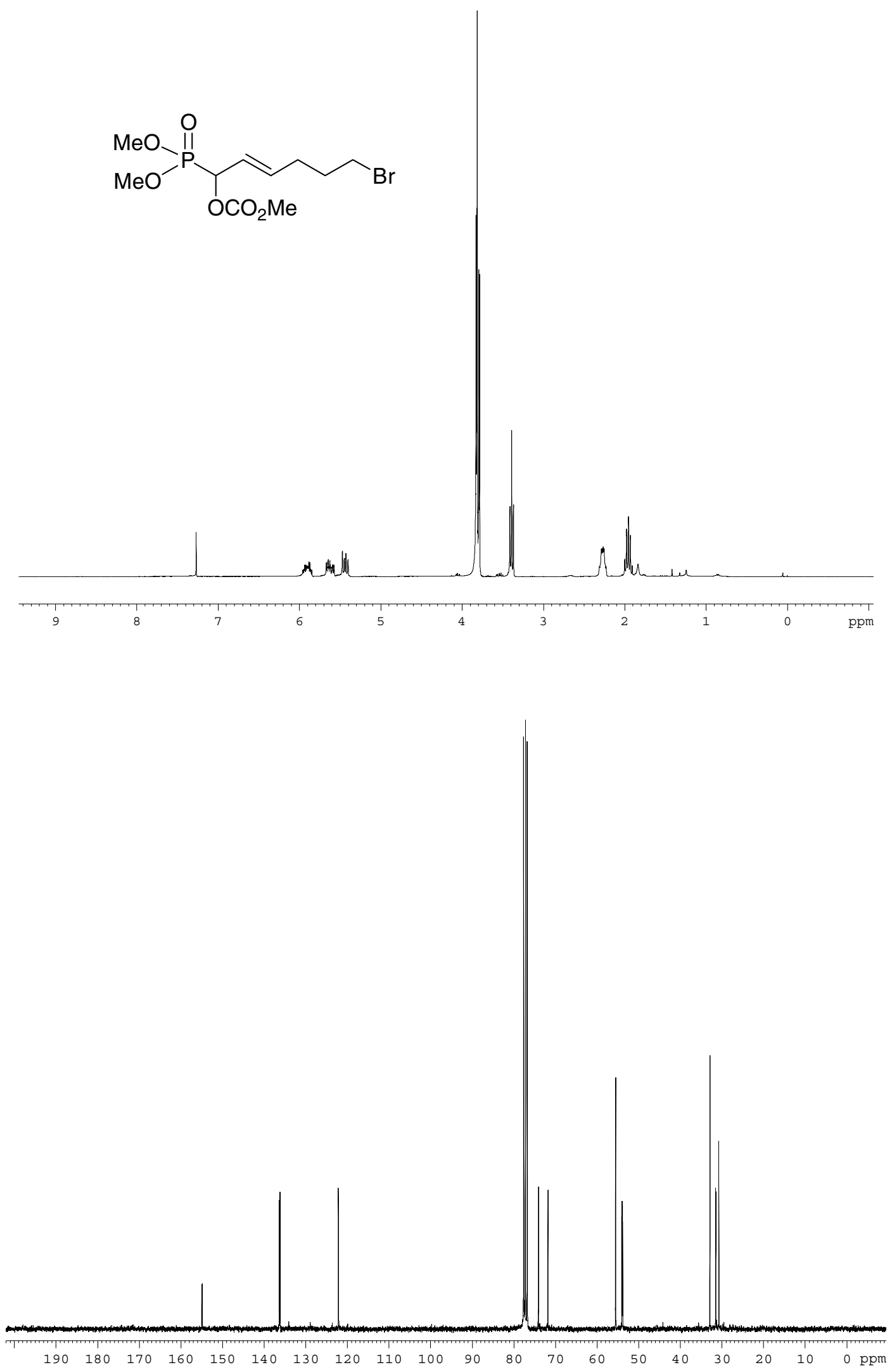

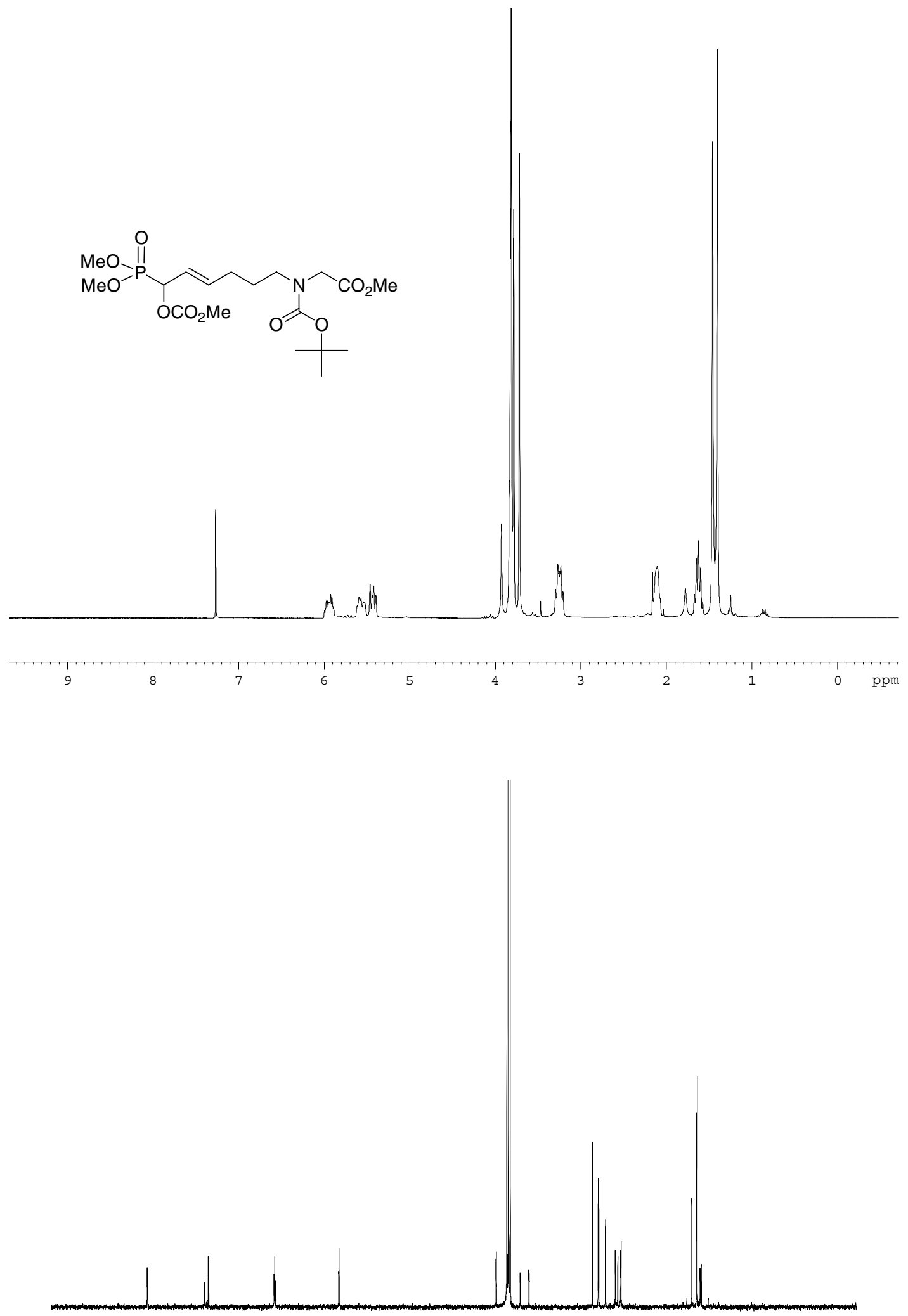

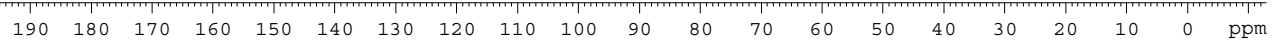




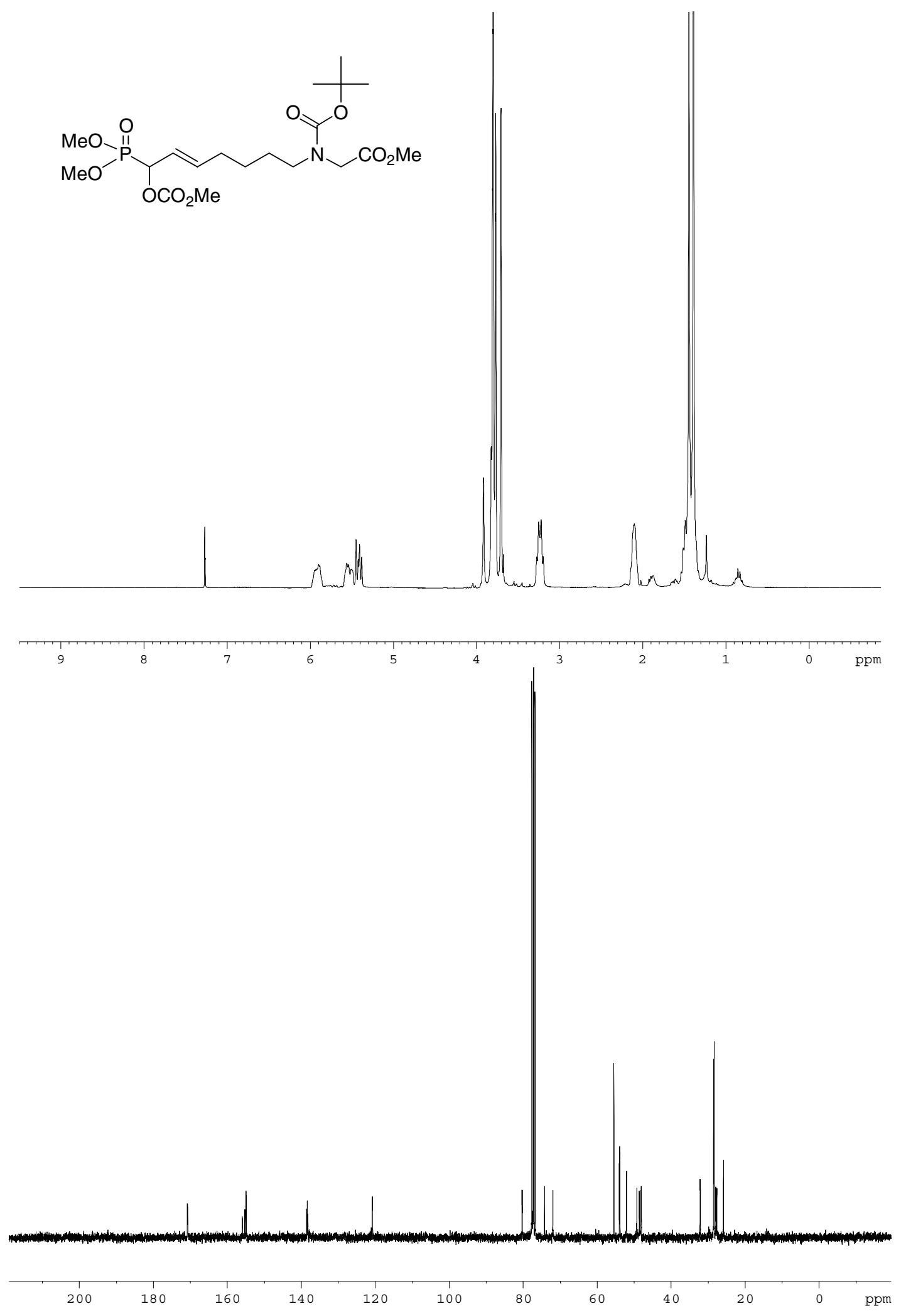



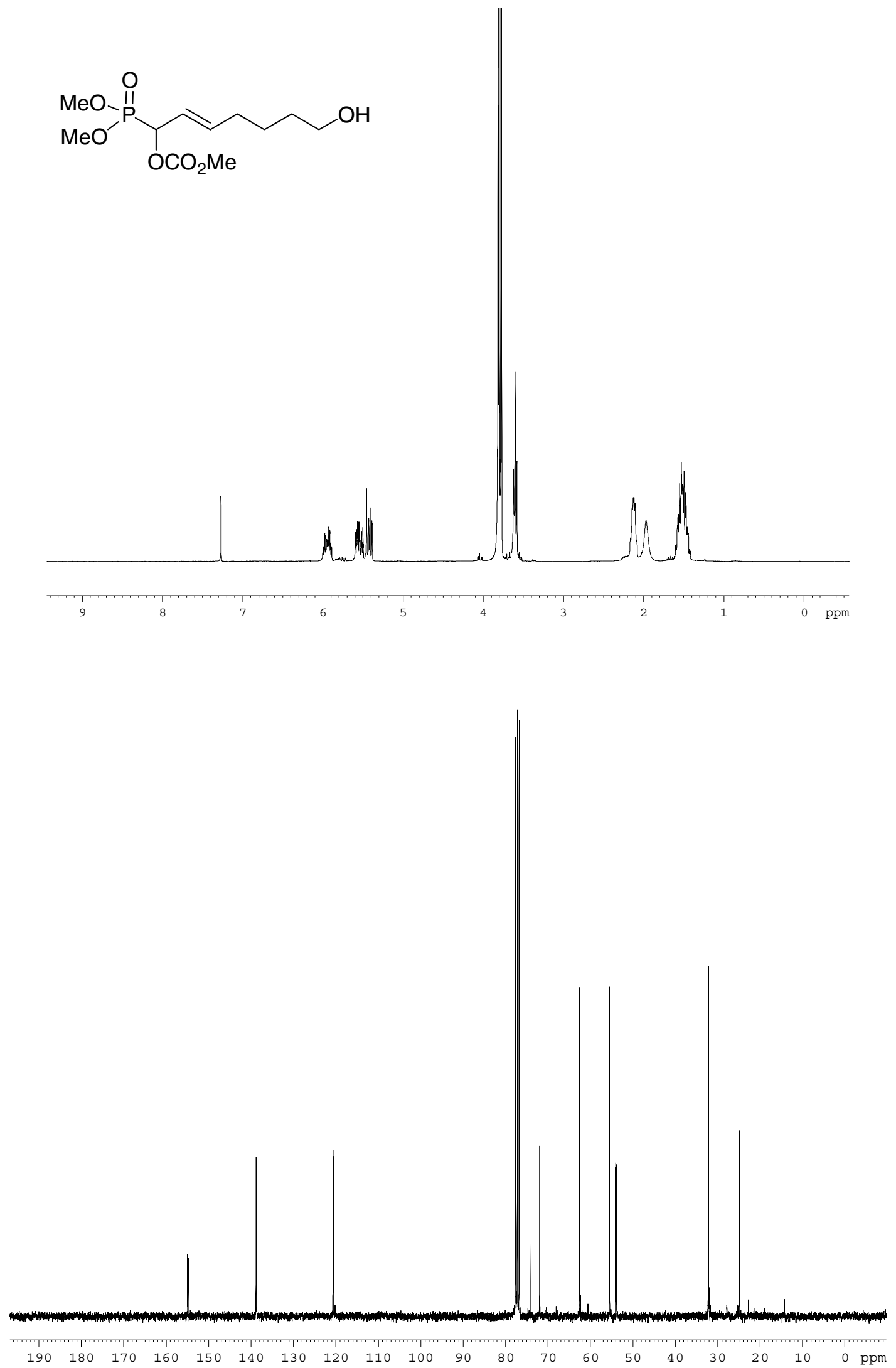


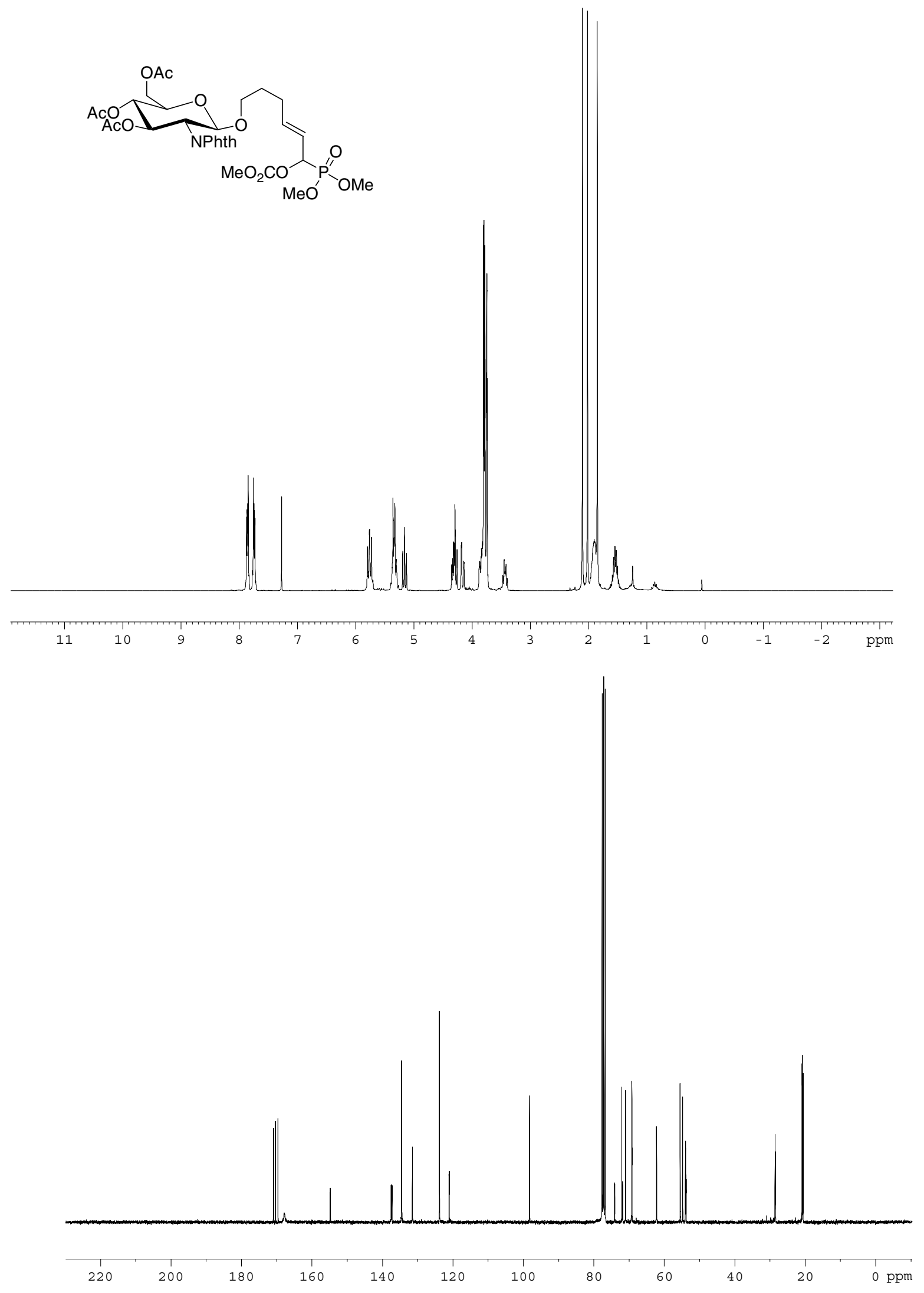




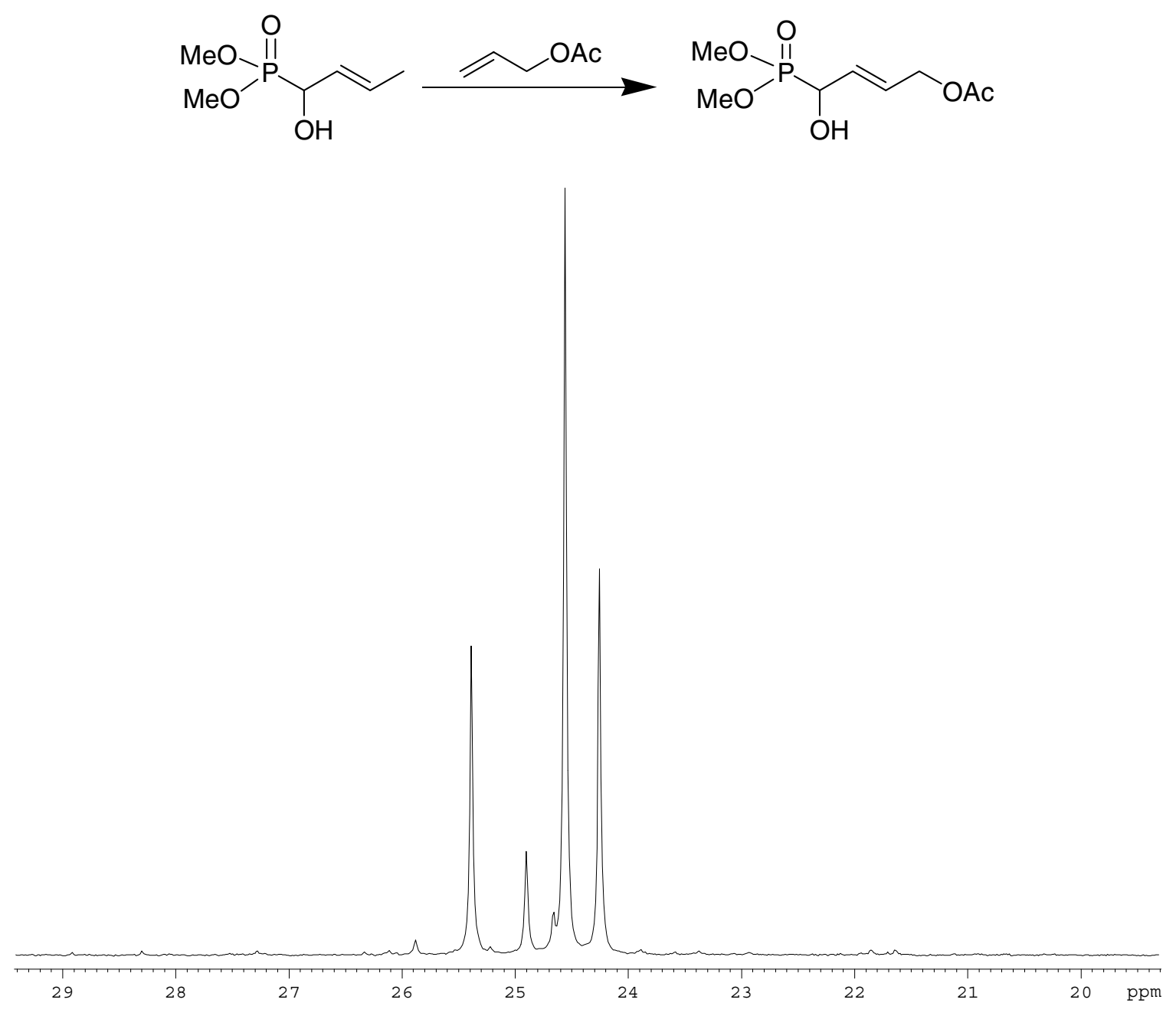


Column-S,S Whelk-O, EtOH:hexane (20:80), $1 \mathrm{~mL} / \mathrm{min}$, det $254 \mathrm{~nm}$

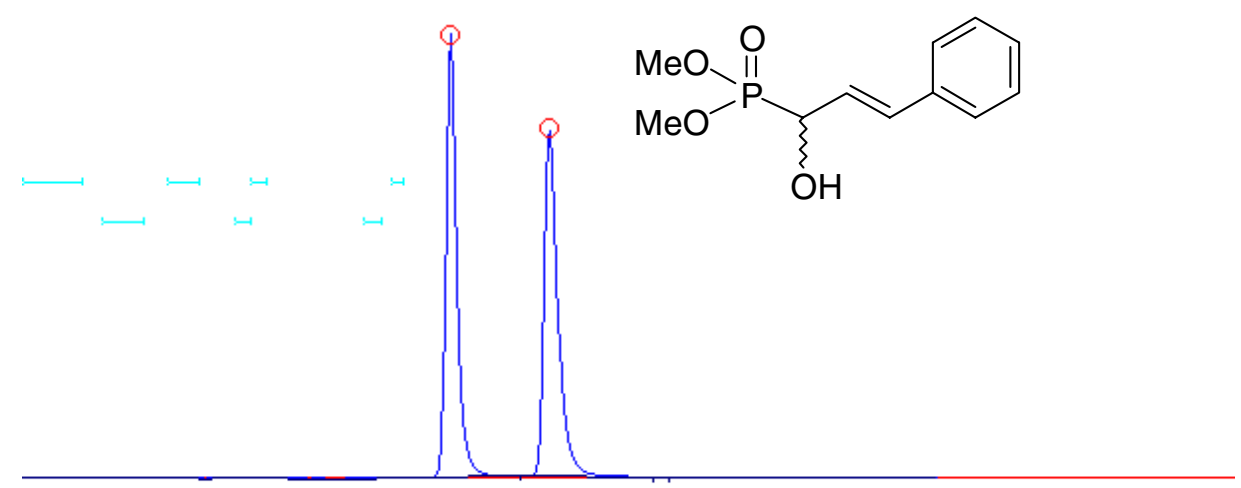

Racemic

\begin{tabular}{|c|c|c|}
\hline $\begin{array}{c}\text { Retention time } \\
(\text { min })\end{array}$ & Area & \% Area \\
\hline 6.97 & 5211 & 49.7 \\
\hline 8.58 & 5281 & 50.3 \\
\hline
\end{tabular}

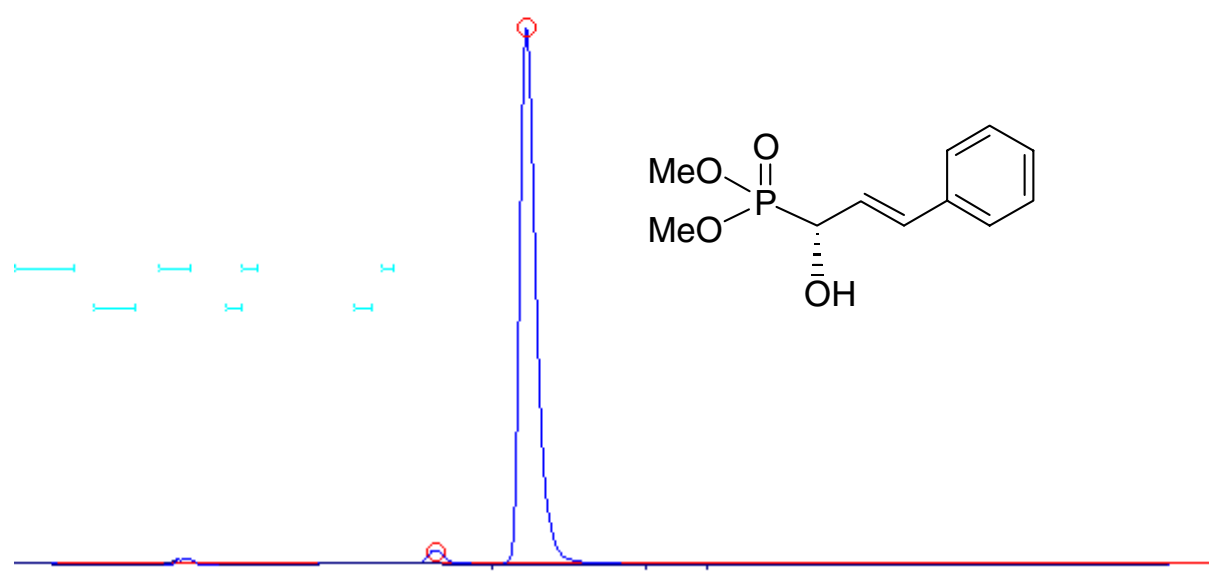

R Isomer (95\% e.e.)

\begin{tabular}{|c|c|c|c|}
\hline $\begin{array}{c}\text { Retention time } \\
(\mathrm{min})\end{array}$ & Area & \% Area & Ratio \\
\hline 6.88 & 503 & 2.5 & 39 \\
\hline 8.38 & 19854 & 97.5 & 1 \\
\hline
\end{tabular}



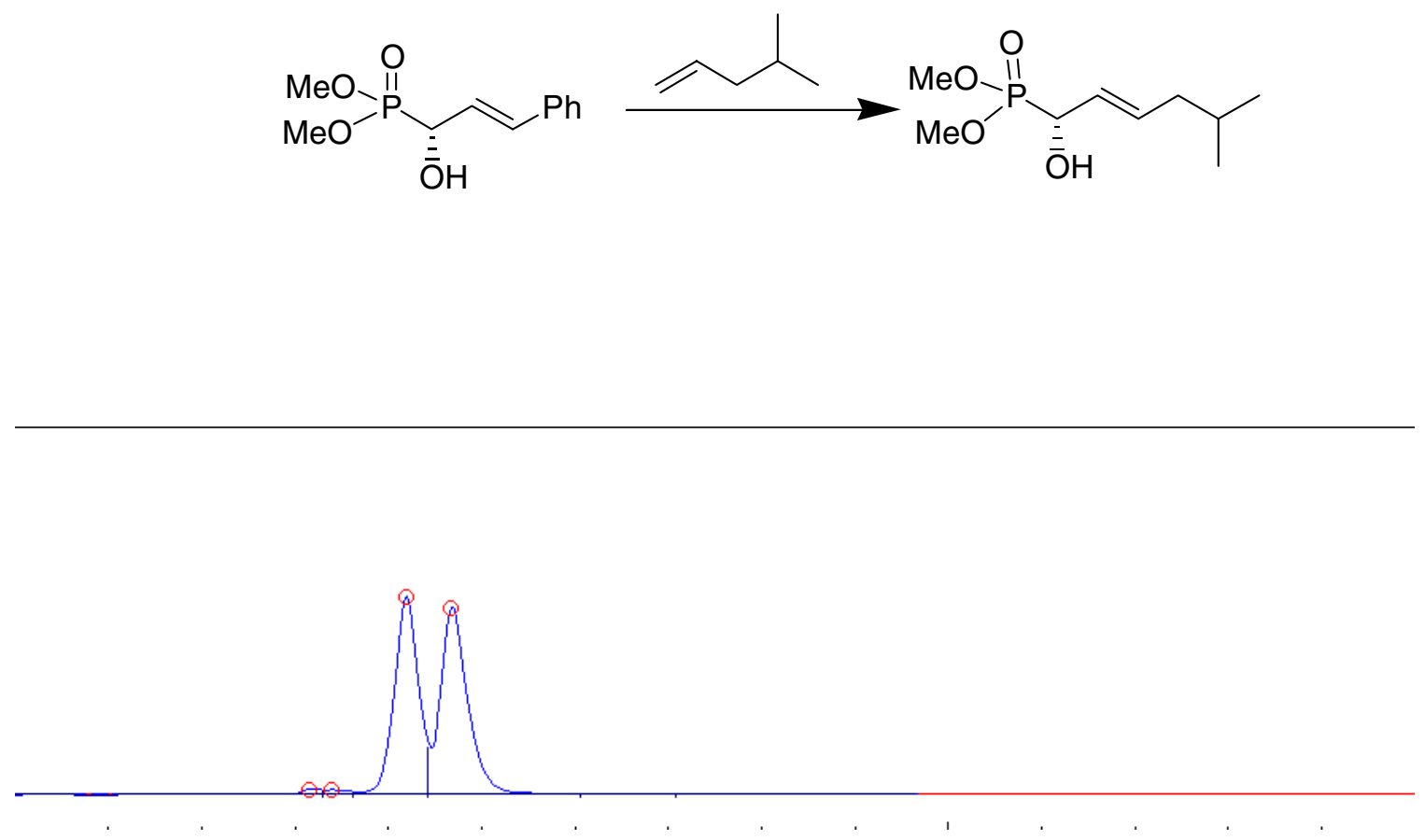

Racemic

\begin{tabular}{|c|c|c|}
\hline $\begin{array}{c}\text { Retention time } \\
(\mathrm{min})\end{array}$ & Area & \% Area \\
\hline 4.17 & 15318 & 48.2 \\
\hline 4.65 & 16481 & 51.8 \\
\hline
\end{tabular}

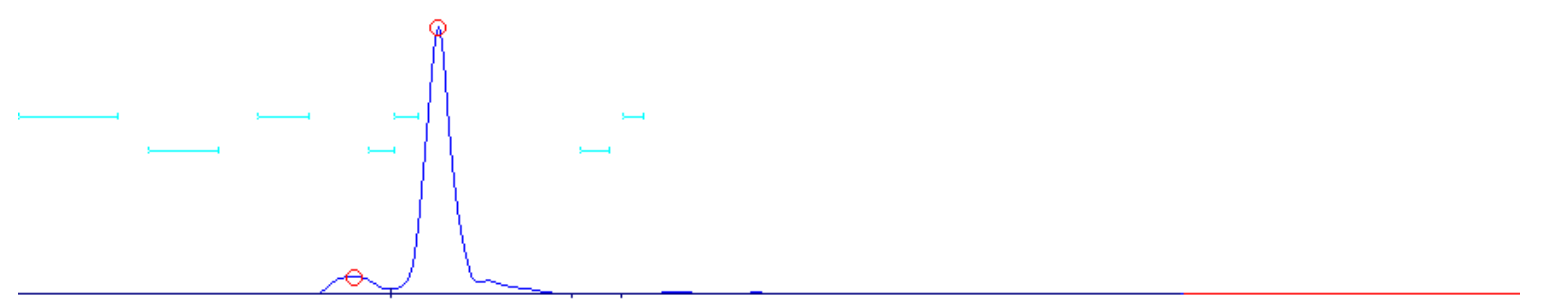

R Isomer (95\% e.e.)

\begin{tabular}{|c|c|}
\hline $\begin{array}{c}\text { Retention time } \\
(\mathrm{min})\end{array}$ & Area \\
\hline 3.5 & impurity \\
\hline 4.17 & 10595 \\
\hline 4.65 & Not measured \\
\hline
\end{tabular}


Column-Chiralpak-AS, EtOH:hexane (20:80), $1 \mathrm{~mL} / \mathrm{min}$, det $210 \mathrm{~nm}$

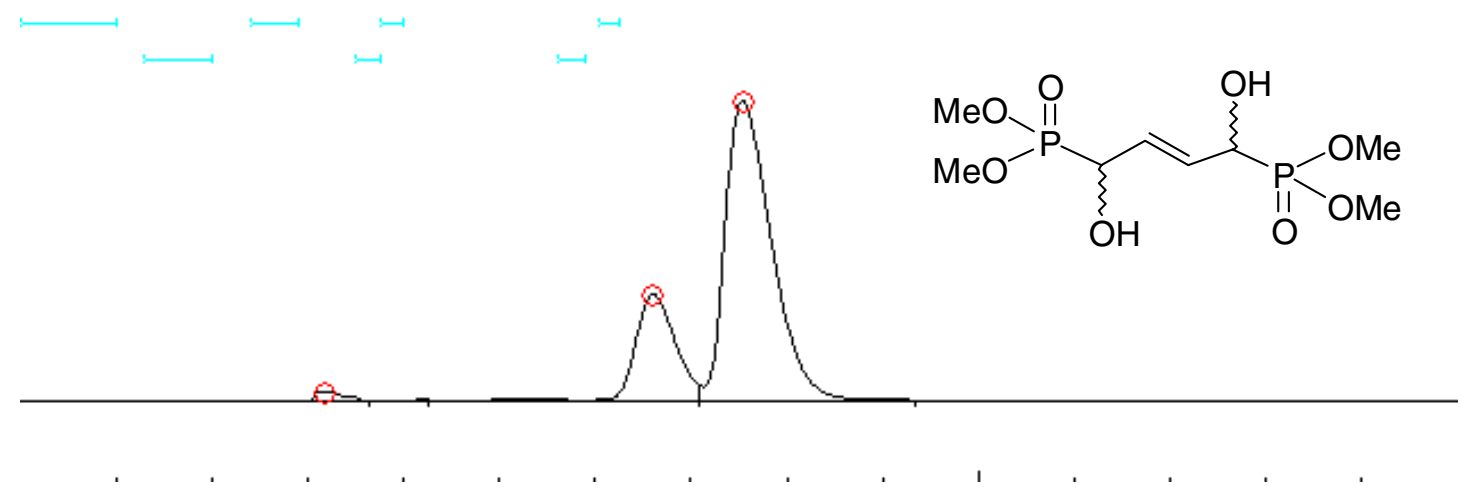

Dimer from Racemic 2a

\begin{tabular}{|c|c|c|}
\hline $\begin{array}{c}\text { Retention time } \\
(\text { min) }\end{array}$ & Area & \% Area \\
\hline 6.57 & 2886 & 23 \\
\hline 7.50 & 9618 & 77 \\
\hline
\end{tabular}

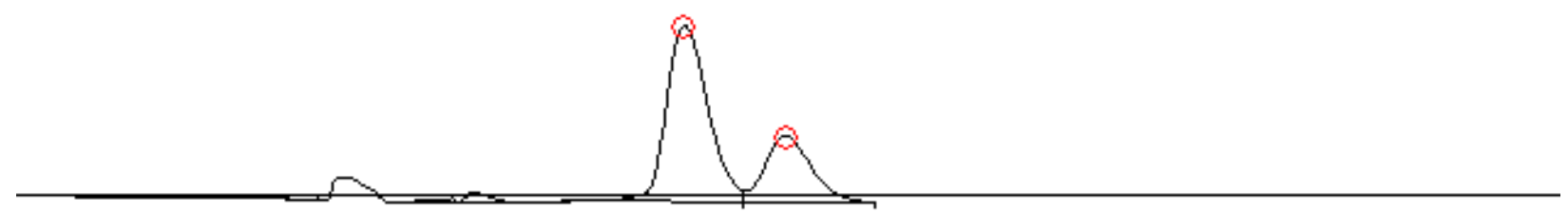

Isolated Crude from Dimerization of R-2a (70\% e.e.)

\begin{tabular}{|c|c|c|}
\hline $\begin{array}{c}\text { Retention time } \\
\text { (min) }\end{array}$ & Area & \% Area \\
\hline 6.45 & 2211 & 75 \\
\hline 7.43 & 728 & 25 \\
\hline
\end{tabular}




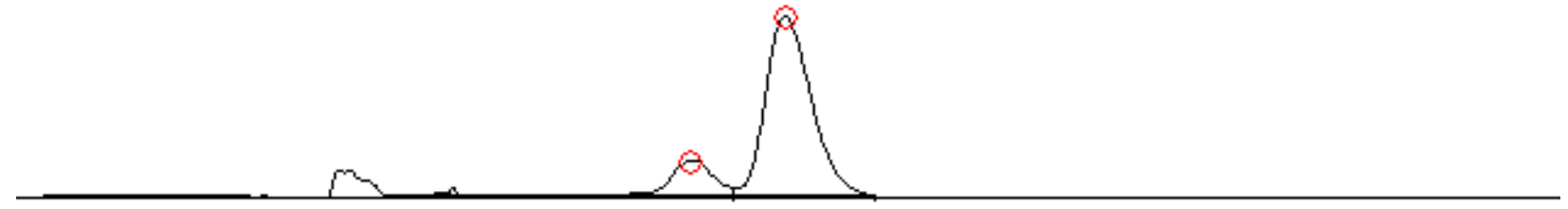

Recrystallized product from Dimerization of R-2a

\begin{tabular}{|c|c|c|}
\hline $\begin{array}{c}\text { Retention time } \\
(\mathrm{min})\end{array}$ & Area & $\%$ Area \\
\hline 6.52 & 462 & 17 \\
\hline 7.43 & 2320 & 83 \\
\hline
\end{tabular}

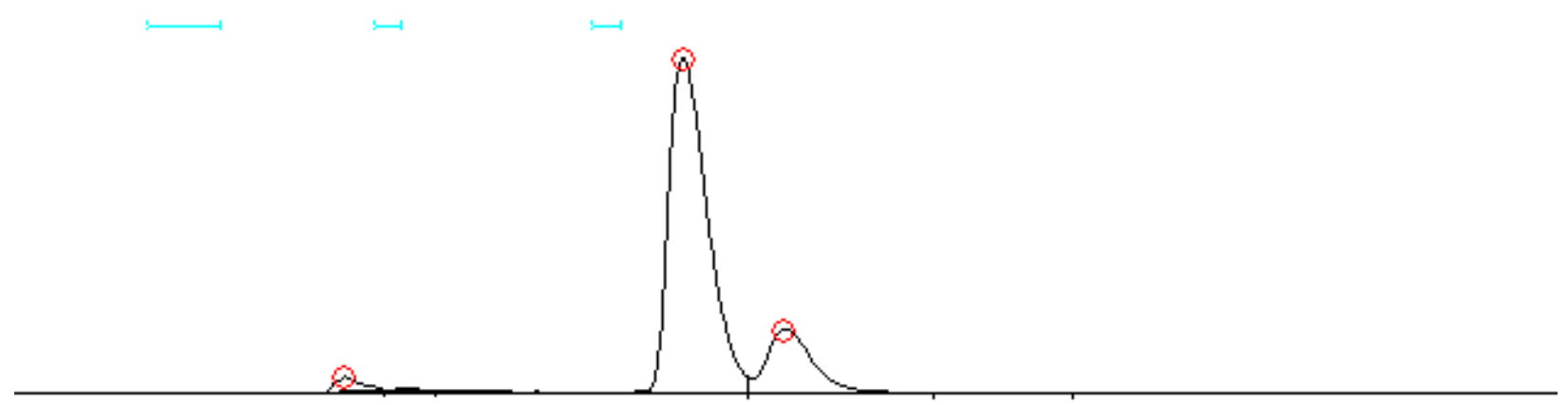

Mother liquor from Recrystallization of Dimerization Product of R-2a

\begin{tabular}{|c|c|c|}
\hline $\begin{array}{c}\text { Retention time } \\
(\mathrm{min})\end{array}$ & Area & $\%$ Area \\
\hline 6.47 & 7834 & 82 \\
\hline 7.45 & 1748 & 18 \\
\hline
\end{tabular}


Table 1. Crystal data and structure refinement for cds14502.

Identification code

s14502/lt

Empirical formula

C8 H18 O8 P2

Formula weight

304.16

Temperature

120(2) K

Wavelength

$0.71073 \AA$

Crystal system

Monoclinic

Space group

$\mathrm{C} 2 / \mathrm{c}$

Unit cell dimensions

$\mathrm{a}=20.1094(14) \AA$

$\alpha=90^{\circ}$.

$\mathrm{b}=7.4334(5) \AA$

$\beta=120.158(4)^{\circ}$.

$\mathrm{c}=10.5575(7) \AA$

$\gamma=90^{\circ}$.

Volume

1364.53(16) $\AA^{3}$

Z

4

Density (calculated)

$1.481 \mathrm{Mg} / \mathrm{m}^{3}$

Absorption coefficient

$0.347 \mathrm{~mm}^{-1}$

$\mathrm{F}(000)$

640

Crystal size

$0.30 \times 0.30 \times 0.28 \mathrm{~mm}^{3}$

Theta range for data collection

2.34 to $29.97^{\circ}$.

Index ranges

$-28 \leq \mathrm{h} \leq 27,-10 \leq \mathrm{k} \leq 10,-14 \leq \mathrm{l} \leq 14$

Reflections collected

16236

Independent reflections

$1882[\mathrm{R}(\mathrm{int})=0.0172]$

Completeness to theta $=29.97^{\circ}$

Absorption correction

$94.4 \%$

Max. and min. transmission

None

Refinement method

0.9091 and 0.9031

Full-matrix least-squares on $\mathrm{F}^{2}$

Data / restraints / parameters

1882 / 0 / 118

Goodness-of-fit on $\mathrm{F}^{2}$

1.099

Final $\mathrm{R}$ indices [I>2sigma(I)]

$\mathrm{R} 1=0.0383, \mathrm{wR} 2=0.1077$

$\mathrm{R}$ indices (all data)

$\mathrm{R} 1=0.0437, \mathrm{wR} 2=0.1125$

Largest diff. peak and hole

0.606 and -0.384 e. $\AA^{-3}$ 
Table 2. Atomic coordinates ( $\mathrm{x} 10^{4}$ ) and equivalent isotropic displacement parameters $\left(\AA^{2} \times 10^{3}\right)$ for cds14502. $U(e q)$ is defined as one third of the trace of the orthogonalized $U^{\mathrm{ij}}$ tensor.

\begin{tabular}{lrrrr}
\hline & $\mathrm{x}$ & $\mathrm{y}$ & $\mathrm{z}$ & $\mathrm{U}(\mathrm{eq})$ \\
\hline $\mathrm{P}(1)$ & $1269(1)$ & $2379(1)$ & $1048(1)$ & $22(1)$ \\
$\mathrm{O}(1)$ & $1262(1)$ & $396(1)$ & $1001(1)$ & $28(1)$ \\
$\mathrm{O}(2)$ & $634(1)$ & $3327(2)$ & $-383(1)$ & $28(1)$ \\
$\mathrm{O}(3)$ & $1129(1)$ & $3158(2)$ & $2284(1)$ & $32(1)$ \\
$\mathrm{O}(4)$ & $2736(1)$ & $2724(2)$ & $2628(1)$ & $30(1)$ \\
$\mathrm{C}(1)$ & $2139(1)$ & $3420(2)$ & $1283(2)$ & $24(1)$ \\
$\mathrm{C}(2)$ & $-164(1)$ & $2796(3)$ & $-993(2)$ & $35(1)$ \\
$\mathrm{C}(3)$ & $1269(2)$ & $2115(3)$ & $3542(2)$ & $48(1)$ \\
$\mathrm{C}(4)$ & $2236(1)$ & $3044(2)$ & $-26(2)$ & $26(1)$ \\
& & & & \\
\hline
\end{tabular}


Table 3. Bond lengths $[\AA ̊]$ and angles $\left[{ }^{\circ}\right]$ for cds 14502 .

\begin{tabular}{lc}
\hline $\mathrm{P}(1)-\mathrm{O}(1)$ & $1.4747(11)$ \\
$\mathrm{P}(1)-\mathrm{O}(2)$ & $1.5719(10)$ \\
$\mathrm{P}(1)-\mathrm{O}(3)$ & $1.5772(11)$ \\
$\mathrm{P}(1)-\mathrm{C}(1)$ & $1.8131(14)$ \\
$\mathrm{O}(2)-\mathrm{C}(2)$ & $1.4522(19)$ \\
$\mathrm{O}(3)-\mathrm{C}(3)$ & $1.438(2)$ \\
$\mathrm{O}(4)-\mathrm{C}(1)$ & $1.4181(17)$ \\
$\mathrm{C}(1)-\mathrm{C}(4)$ & $1.5146(19)$ \\
$\mathrm{C}(4)-\mathrm{C}(4) \# 1$ & $1.315(3)$ \\
& \\
$\mathrm{O}(1)-\mathrm{P}(1)-\mathrm{O}(2)$ & $115.23(6)$ \\
$\mathrm{O}(1)-\mathrm{P}(1)-\mathrm{O}(3)$ & $113.12(6)$ \\
$\mathrm{O}(2)-\mathrm{P}(1)-\mathrm{O}(3)$ & $103.54(6)$ \\
$\mathrm{O}(1)-\mathrm{P}(1)-\mathrm{C}(1)$ & $115.01(6)$ \\
$\mathrm{O}(2)-\mathrm{P}(1)-\mathrm{C}(1)$ & $101.69(6)$ \\
$\mathrm{O}(3)-\mathrm{P}(1)-\mathrm{C}(1)$ & $106.96(6)$ \\
$\mathrm{C}(2)-\mathrm{O}(2)-\mathrm{P}(1)$ & $119.19(10)$ \\
$\mathrm{C}(3)-\mathrm{O}(3)-\mathrm{P}(1)$ & $122.17(12)$ \\
$\mathrm{O}(4)-\mathrm{C}(1)-\mathrm{C}(4)$ & $114.49(12)$ \\
$\mathrm{O}(4)-\mathrm{C}(1)-\mathrm{P}(1)$ & $104.72(9)$ \\
$\mathrm{C}(4)-\mathrm{C}(1)-\mathrm{P}(1)$ & $110.70(9)$ \\
$\mathrm{C}(4) \# 1-\mathrm{C}(4)-\mathrm{C}(1)$ & $123.18(17)$ \\
\hline
\end{tabular}

Symmetry transformations used to generate equivalent atoms:

$\# 1-x+1 / 2,-y+1 / 2,-z$ 
Table 4. Anisotropic displacement parameters $\left(\AA^{2} \times 10^{3}\right)$ for cds 14502 . The anisotropic displacement factor exponent takes the form: $-2 \pi^{2}\left[h^{2} a^{* 2} U^{11}+\ldots+2 h k a^{*} b^{*} U^{12}\right]$

\begin{tabular}{lllllll}
\hline & $\mathrm{U}^{11}$ & $\mathrm{U}^{22}$ & $\mathrm{U}^{33}$ & $\mathrm{U}^{23}$ & $\mathrm{U}^{13}$ & $\mathrm{U}^{12}$ \\
\hline $\mathrm{P}(1)$ & $24(1)$ & $22(1)$ & $21(1)$ & $1(1)$ & $12(1)$ & $1(1)$ \\
$\mathrm{O}(1)$ & $28(1)$ & $22(1)$ & $33(1)$ & $0(1)$ & $14(1)$ & $-1(1)$ \\
$\mathrm{O}(2)$ & $25(1)$ & $33(1)$ & $25(1)$ & $6(1)$ & $12(1)$ & $2(1)$ \\
$\mathrm{O}(3)$ & $44(1)$ & $31(1)$ & $29(1)$ & $4(1)$ & $24(1)$ & $6(1)$ \\
$\mathrm{O}(4)$ & $29(1)$ & $28(1)$ & $25(1)$ & $2(1)$ & $7(1)$ & $-4(1)$ \\
$\mathrm{C}(1)$ & $26(1)$ & $24(1)$ & $22(1)$ & $-1(1)$ & $11(1)$ & $-2(1)$ \\
$\mathrm{C}(2)$ & $23(1)$ & $44(1)$ & $33(1)$ & $4(1)$ & $12(1)$ & $1(1)$ \\
$\mathrm{C}(3)$ & $72(2)$ & $52(1)$ & $30(1)$ & $9(1)$ & $34(1)$ & $5(1)$ \\
$\mathrm{C}(4)$ & $28(1)$ & $26(1)$ & $24(1)$ & $0(1)$ & $13(1)$ & $-4(1)$ \\
& & & & & & \\
\hline
\end{tabular}


Table 5. Hydrogen coordinates ( $\times 10^{4}$ ) and isotropic displacement parameters $\left(\AA^{2} \times 10^{3}\right)$ for cds 14502 .

\begin{tabular}{lcccc}
\hline & $\mathrm{x}$ & $\mathrm{y}$ & $\mathrm{z}$ & $\mathrm{U}(\mathrm{eq})$ \\
& & & & \\
$\mathrm{H}(1)$ & $2057(10)$ & $4840(20)$ & $1194(19)$ & $23(4)$ \\
$\mathrm{H}(2 \mathrm{~A})$ & $-190(15)$ & $1650(30)$ & $-1110(30)$ & $54(7)$ \\
$\mathrm{H}(2 \mathrm{~B})$ & $-445(16)$ & $3430(30)$ & $-1850(30)$ & $62(7)$ \\
$\mathrm{H}(2 \mathrm{C})$ & $-337(16)$ & $2890(40)$ & $-250(30)$ & $61(7)$ \\
$\mathrm{H}(3 \mathrm{~A})$ & $1010(30)$ & $2630(50)$ & $3840(50)$ & $118(15)$ \\
$\mathrm{H}(3 \mathrm{~B})$ & $1230(20)$ & $860(50)$ & $3390(40)$ & $101(11)$ \\
$\mathrm{H}(3 \mathrm{C})$ & $1780(30)$ & $2350(50)$ & $4260(50)$ & $130(18)$ \\
$\mathrm{H}(4)$ & $1865(12)$ & $3640(30)$ & $-980(20)$ & $36(5)$ \\
$\mathrm{H}(4 \mathrm{~A})$ & $3073(16)$ & $3600(40)$ & $3130(30)$ & $61(7)$ \\
& & & & \\
\hline
\end{tabular}


Projection view with 50\% thermal ellipsoids

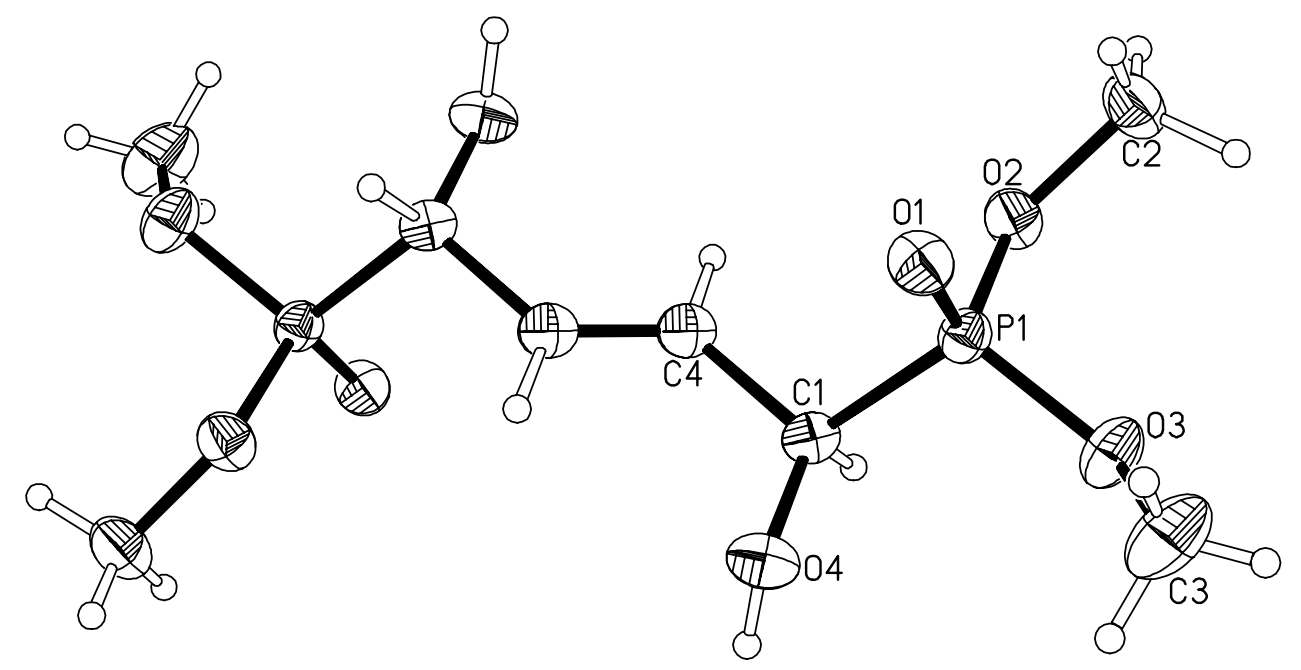

Crystal Packing with H-bonds:

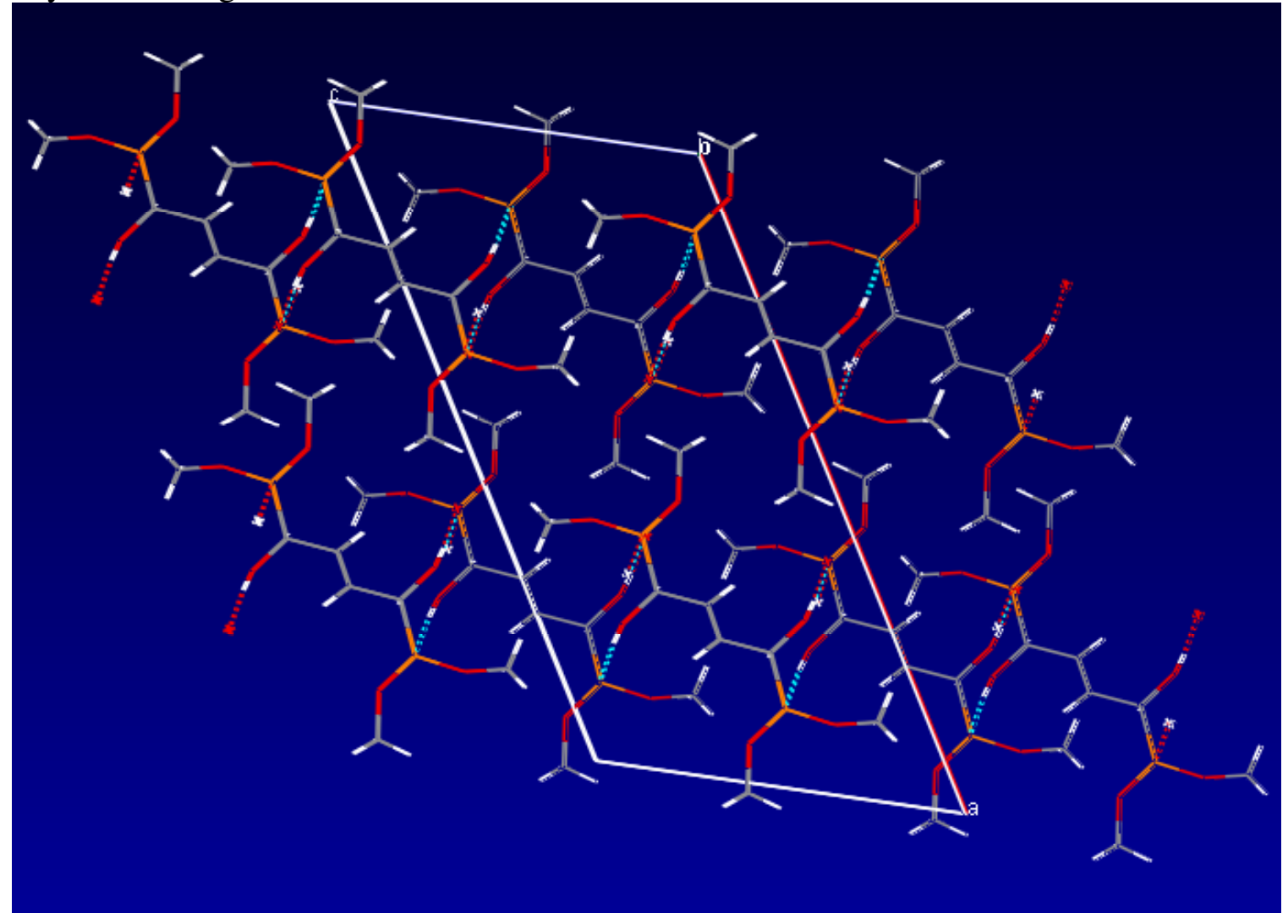

